\title{
Estimation of Cirrus and Stratus Cloud Heights Using Landsat Imagery
}

\author{
Yasushi Inomata, ${ }^{*}$ R. E. Feind, and R. M. Welch \\ Institute of Atmospheric Sciences, South Dakota School of Mines and Technology, Rapid City, South Dakota
}

(Manuscript received 7 February 1995, in final form 20 August 1995)

ABSTRACT

A new method based upon high-spatial-resolution imagery is presented that matches cloud and shadow regions to estimate cirrus and stratus cloud heights. The distance between the cloud and the matching shadow pattern is accomplished using the $2 \mathrm{D}$ cross-correlation function from which the cloud height is derived. The distance between the matching cloud-shadow patterns is verified manually. The derived heights also are validated through comparison with a temperature-based retrieval of cloud height. It is also demonstrated that an estimate of cloud thickness can be retrieved if both the sunside and antisunside of the cloud-shadow pair are apparent. The technique requires some intepretation to determine the cloud height level retrieved (i.e., the top, base, or midlevel). It is concluded that the method is accurate to within several pixels, equivalent to cloud height variations of about $\pm 250 \mathrm{~m}$. The results show that precise placement of the templates is unnecessary, so that the development of a semiautomated procedure is possible. Cloud templates of about 64 pixels on a side or larger produce consistent results. The procedure was repeated for imagery degraded to simulate lower spatial resolutions. The results suggest that spatial resolution of $150-200 \mathrm{~m}$ or better is necessary in order to obtain stable cloud height retrievals.

\section{Introduction}

A thorough description and understanding of processes at the earth's surface and in the atmosphere are necessary before realistic climate predictions can be realized. Important large-scale cloud parameters include: cloud-top and cloud-base height, cloud optical thickness, cloud effective particle size and phase, liquid or ice content, cloud cover, and cloud morphology. Roeckner et al. (1987) suggest that global warming may significantly alter cloud optical properties, particularly for high clouds, and Mitchell et al. (1989) suggest that significant adjustments in the water-ice ratio in mixed phase clouds may result from global warming. In this case, some ice clouds would be replaced by water clouds, thereby altering the balance between solar reflection and infrared emission.

Cirrus clouds are characterized by relatively low albedos and low cloud-top temperatures. Generally, depending upon their optical thickness, these clouds have a warming effect on climate. Based upon 12 years of ship-reported observations over the north

\footnotetext{
* Current affiliation: Department of Electric Engineering, Kitakyushu National College of Technology, 5-20-1 Shii, Kokuraminami, Kitakyushu, 803 Japan.
}

Corresponding author address: Dr. R. M. Welch, Institute of Atmospheric Sciences, South Dakota School of Mines and Technology, 501 East St. Joseph Street, Rapid City, SD 57701-3995.
Atlantic Ocean, Hahn et al. (1982) report cirrus cloudiness with a frequency of occurrence of $20 \%-$ $45 \%$, depending upon location and season. The First ISCCP (International Satellite Cloud Climatology Project) Regional Experiments (FIRE) have improved our understanding of cirrus cloudiness, especially cloud amount, height, and optical depth. The importance of small ice crystals and the superiority of hexagonal crystal scattering phase functions over spheres have been established. However, global cloud parameters remain largely unknown.

Two critical cloud parameters are cloud-top and cloud-base heights; cloud-base heights strongly modulate downward longwave irradiances at the earth's surface (Gupta 1989). Likewise, the presence of cloud shadows significantly alters the downward solar irradiances at the earth's surface and contaminates the reflected signatures used for remote sensing applications. While cloud-top height can be directly estimated from satellite infrared channel sensors, cloud-base height must be inferred. Frouin et al. (1988) used the combination of TOVS sounder profiles and GOES cloud parameters to deduce cloud-base height from cloud-top height and liquid water path. Four different methods were examined; it was found that the simpler methods often performed as well as some of the more complex approaches. Pandey et al. (1983), Wu (1987), and Minnis et al. (1993) used microwave and infrared satellite measurements to infer cloud-base heights, and Manschke (1988) showed that cloud-base temperature 

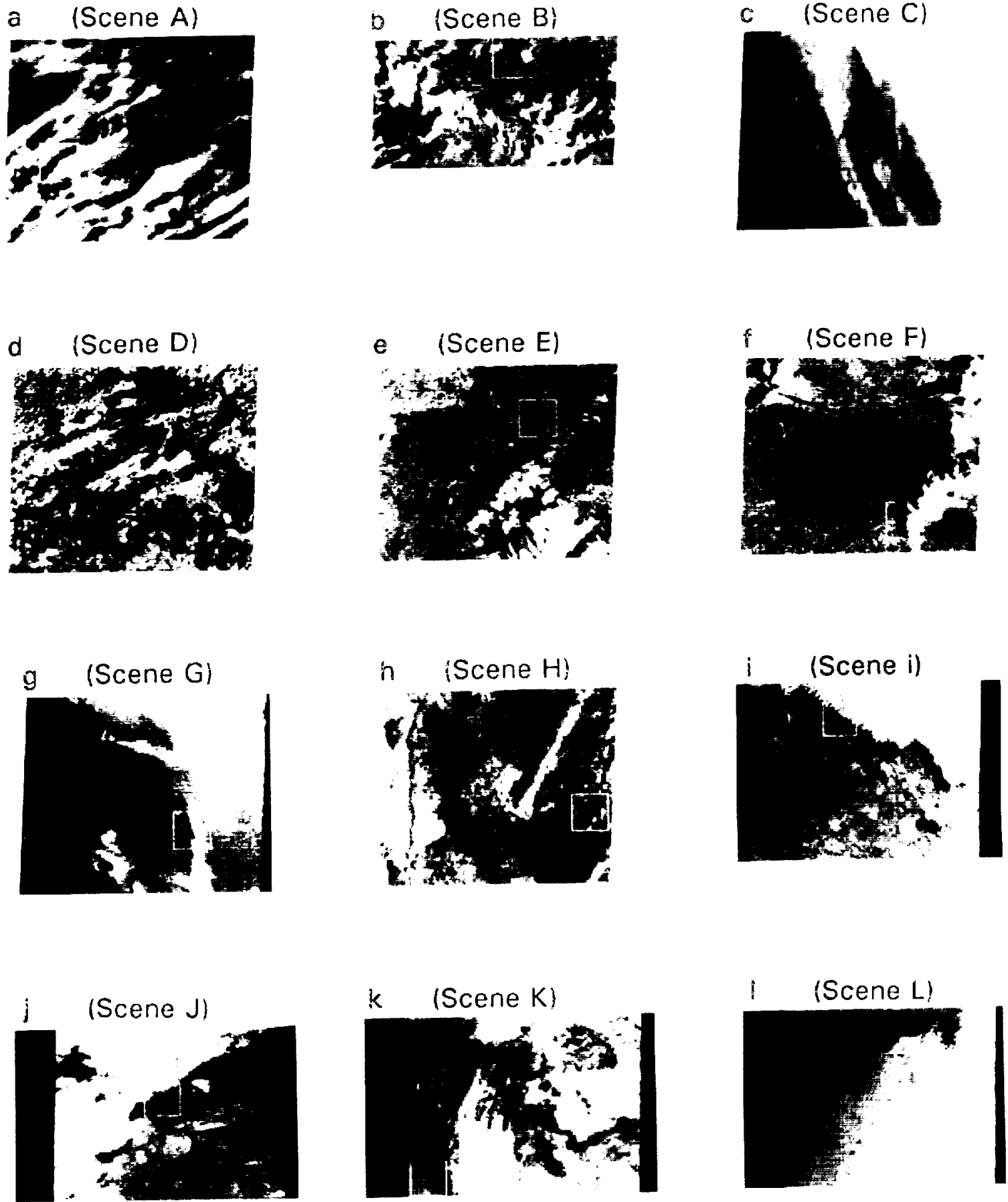

FIG. I. (a)-(1) Eight Landsat MSS scenes (A)-(H) and the four TM scenes (I)-(L) used in this study. Each MSS scene is 185 $\mathrm{km}$ wide by $170 \mathrm{~km}$; each TM scene is one-quarter this size. A description of each scene is given in Table 1 . The white-black highlighted boxes shown are the $512 \times 512$ pixel subregions used in the analysis.

can be retrieved from AVHRR data in convective situations.

Using high-spatial-resolution imagery, Gurney (1982) and Hambrick et al. (1987) showed the feasibility of locating cloud shadows, and Berendes et al.
(1992) developed an automated technique to estimate cumulus cloud-base height. The purpose of the present investigation is to demonstrate that high-spatial-resolution imagery likewise can be used to provide an independent measurement of cirrus and stratus cloud 
TABLE: 1. Description of 13 Landsat scenes used in this study.

\begin{tabular}{|c|c|c|c|c|c|c|c|c|c|}
\hline Scene & Type & Landsat & Path/Row & Lat, Long & Date & $\begin{array}{c}\text { Solar } \\
\text { zenith }\end{array}$ & $\begin{array}{c}\text { Solar } \\
\text { azimuth }\end{array}$ & $\begin{array}{l}\text { Skew } \\
\text { angle }\end{array}$ & Location \\
\hline A & MSS & 3 & $125 / 31$ & $41^{\circ} 37^{\prime} \mathrm{N}, 127^{\circ} 50^{\prime} \mathrm{E}$ & 20 Aug 79 & $40^{\circ}$ & $128^{\circ}$ & $12.2^{\circ}$ & China \\
\hline B & MSS & 2 & $36 / 29$ & $44^{\circ} 29^{\prime} \mathrm{N}, 103^{\circ} 34^{\prime} \mathrm{W}$ & 20 May 81 & $34^{\circ}$ & $127^{\circ}$ & $12.8^{\circ}$ & South Dakota \\
\hline $\mathrm{C}$ & MSS & 5 & $28 / 38$ & $31^{\circ} 45^{\prime} \mathrm{N}, 99^{\circ} 04^{\prime} \mathrm{W}$ & 28 Oct 85 & $52^{\circ}$ & $147^{\circ}$ & $9.7^{\circ}$ & Texas \\
\hline D & MSS & 4 & $161 / 25$ & $50^{\circ} 17^{\prime} \mathrm{N}, 61^{\circ} 36^{\prime} \mathrm{E}$ & $17 \operatorname{Sep} 89$ & $52^{\circ}$ & $151^{\circ}$ & $12.9^{\circ}$ & Russia \\
\hline $\mathbf{E}$ & MSS & 4 & $31 / 31$ & $41^{\circ} 46^{\prime} \mathrm{N}, 100^{\circ} 43^{\prime} \mathrm{W}$ & 18 Sep 89 & $46^{\circ}$ & $144^{\circ}$ & $11.0^{\circ}$ & Nebraska \\
\hline $\mathbf{F}$ & MSS & 4 & $30 / 32$ & $40^{\circ} 20^{\prime} \mathrm{N}, 99^{\circ} 38^{\prime} \mathrm{W}$ & 27 Oct 89 & $48^{\circ}$ & $146^{\circ}$ & $10.8^{\circ}$ & Nebraska \\
\hline $\mathrm{G}$ & MSS & 5 & $23 / 27$ & $47^{\circ} 27^{\prime} \mathrm{N}, 86^{\circ} 27^{\prime} \mathrm{W}$ & 18 Oct 89 & $51^{\circ}$ & $146^{\circ}$ & $12.2^{\circ}$ & Lake Superior \\
\hline $\mathbf{H}$ & MSS & 5 & $33 / 36$ & $34^{\circ} 37^{\prime} \mathrm{N}, 106^{\circ} 00^{\prime} \mathrm{W}$ & 16 Nov 85 & $59^{\circ}$ & $151^{\circ}$ & $10.0^{\circ}$ & New Mexico \\
\hline I & TM & 4 & $207 / 110$ & $70^{\circ} 30^{\prime} \mathrm{S}, 27^{\circ} 00^{\prime} \mathrm{E}$ & 7 Feb 89 & $67^{\circ}$ & $68^{\circ}$ & $25.2^{\circ}$ & Antarctica \\
\hline $\mathbf{J}$ & TM & 4 & $215 / 106$ & $65^{\circ} 22^{\prime} \mathrm{S}, 56^{\circ} 55^{\prime} \mathrm{W}$ & 19 Mar 89 & $76^{\circ}$ & $56^{\circ}$ & $20.0^{\circ}$ & Antarctica \\
\hline $\mathbf{K}$ & TM & 4 & $128 / 109$ & $68^{\circ} 48^{\prime} \mathrm{S}, 73^{\circ} 58^{\prime} \mathrm{E}$ & 18 Mar 89 & $78^{\circ}$ & $59^{\circ}$ & $23.2^{\circ}$ & Antarctica \\
\hline $\mathrm{L}$ & TM & 4 & $118 / 107$ & $66^{\circ} 45^{\prime} \mathrm{S}, 91^{\circ} 40^{\prime} \mathrm{E}$ & $12 \operatorname{Mar} 89$ & $75^{\circ}$ & $59^{\circ}$ & $21.1^{\circ}$ & Antarctica \\
\hline $\mathbf{M}$ & $\mathbf{T M}$ & 5 & $025 / 039$ & $31^{\circ} 45^{\prime} \mathrm{N}, 94^{\circ} 23^{\prime} \mathrm{W}$ & 14 Jun 84 & $28^{\circ}$ & $97^{\circ}$ & $9.7^{\circ}$ & Texas \\
\hline
\end{tabular}

heights. Section 2 discusses the Landsat TM data and the scenes used in this study, and section 3 outlines the methodology. Section 4 presents the results, and section 5 concludes.

\section{Data}

Eight Landsat Multispectral Scanner (MSS) and five Thematic Mapper (TM) images are used in this definition-phase study. The eight MSS images and four of the TM images are shown in Fig. 1. The fifth TM image appears in Fig. 9. These images are selected from an archive of Landsat scenes at the South Dakota School of Mines and Technology (SDSM\&T). They are not representative of the full range of cirrus and stratus conditions; however, they represent a variety of cloud types and solar-illumination conditions.

The Landsat satellites are in sun-synchronous orbits, sampling the earth at approximately 0930-1000 local solar time. Each geometrically corrected MSS scene is a $3246 \times 2983$ pixel array covering a surface area of $185 \mathrm{~km} \times 170 \mathrm{~km}$ with pixel resolution of $57 \mathrm{~m}$ in four narrow spectral bands at $0.5-0.6,0.6-0.7,0.7-0.8$, and $0.8-1.1 \mu \mathrm{m}$. Scene B in Fig. 1 is incomplete due to missing data. The gray levels in MSS band 3 have a range from 0 to 127 , corresponding to a saturated reflectance of about 57\% (Robinove 1982). MSS band $I$ is avoided due to greater Rayleigh scattering, and band 4 is avoided due to water vapor absorption. Therefore, MSS bands 2 or 3 can be used for this study. Band 2 has higher Rayleigh scattering, whereas band 3 has oxygen absorption. In this study, MSS band 3 is selected. The TM quad-scenes each cover one-quarter of the area of an MSS scene, but with pixel resolution of $28.5 \mathrm{~m}$ in six visible-near-IR channels at $0.45-0.52$, $0.52-0.60,0.63-0.69,0.76-0.90,1.55-1.75$, and $2.08-2.35 \mu \mathrm{m}$, and in one $114-\mathrm{m}$ spatial resolution thermal channel at $10.4-12.5 \mu \mathrm{m}$. Four of the TM images (i.e., I, J, K, and L from Fig. 1) were obtained over Antarctica, and the fifth image, scene M, (i.e., Fig.
9) was obtained over eastern Texas. TM channel 5, which is digitized over a range of $0-255$, is used in this study because it allows the cloud shadows to be easily distinguished on snow-ice surfaces. Table 1 gives a description of each of the scenes shown in Figs. 1 and 9. From each scene in Fig. 1, subregions of 512 $\times 512$ pixels are chosen and analyzed, as indicated by the white boxes in Fig. 1. A subregion of $750 \times 750$ pixels is chosen from scene $M$ in Fig. 9 for analysis.

\section{Methodology}

The cloud-base height approach developed by Berendes et al. (1992) employed a variety of image processing techniques to identify and then to match cloud edges with their corresponding shadow edges. Cloudbase height was estimated by computing the separation distance between the corresponding generalized Hough transform reference points. While such an approach is applicable for cumulus clouds, in general it cannot be used for clouds with ill-defined edges such as cirrus or stratus. In particular, cirrus clouds often are optically thin and transparent, making edge detection problematical. The precise location of cirrus shadow edges also is extremely difficult over a land surface of variable surface reflectance. Therefore, a much different approach is required.

In many image processing applications, a pixel-bypixel comparison of two images is necessary in order to spatially register them. In this way, it is possible to correct for translational shifts, scale differences, and rotational differences. The typical approach is to compute the normalized cross-correlation function between the two images and to find the translational offset from the correlation function peak. Let $C(x, y)$ be the gray scale (i.e., brightness) value of a portion of a digital image of $M$ columns and $N$ rows, where $x$ and $y$ are the column and row indices, respectively. Now we wish to determine if it contains a subregion that is similar to some subregion $S(x, y)$ of size $J \times K$, where $J \leqslant M$ 
and $K \leqslant N$. Because the correlation function is sensitive to amplitude variations, we first compute the average gray level in the subregions $\bar{C}$ and $\bar{S}$, respectively. Then the correlation function is defined as

$$
R(m, n)=\frac{\sum_{x} \sum_{y}[C(x, y)-\bar{C}]}{\times[S(x-m, y-n)-\bar{S}]},
$$

where the summations are taken over the indices common to both $C(x, y)$ and $S(x, y)$. The correlation function, $R(m, n)$, ranges in value from -1 to 1 . Generally, the values of $m$ and $n$ that maximize the function determine the coordinates in which $S(x, y)$ best matches $C(x, y)$.

A variation of the above image registration procedure using the correlation function is used in this investigation; the $C(x, y)$ region contains the cloud; and subregion $S(x, y)$ contains the shadow region that best matches the cloudy region. Since cloudy regions generally are brighter than the background regions, and since shadow regions generally are darker than the background regions, the values of $m$ and $n$ that minimize the correlation function, $R(m, n)$, provide the best template match in this case. Three techniques are implemented to improve the correlation results. First, in order to enhance the brightness contrast in the image, a histogram equalization (e.g., Richards 1993) is applied to extend the gray-level brightness range of the images. Second, in the cloud subregions, an inversion of the gray scale is applied,

$$
g^{\prime}=255-g,
$$

where $g$ is the original histogram equalized gray level and $g^{\prime}$ is the inverted value. Using this latter transformation for the $C(x, y)$ subregion, then a maximum value of the correlation function is found. In the present case, a $M \times N$ cloudy subregion first is identified. Then a template of the same size is used to search regions suspected of containing the cloud shadow, and the correlation function is generated. From the minimum value (or maximum value if inversion has been applied) of the correlation function, the retrieved values of $m$ and $n$ [Eq. (1)] then provide the horizontal distance $D$ between cloud and shadow in the image (Fig. 2 a),

$$
D=R\left(m^{2}+n^{2}\right)^{1 / 2}
$$

where spatial resolution $R$ is $57 \mathrm{~m}$ for geometrically corrected Landsat MSS data and $28.5 \mathrm{~m}$ for TM data. Cloud top or base, $H$, is computed from solar zenith angle $\theta_{n}$ (Fig. $2 \mathrm{~b}$ ), as

$$
H=D \cot \theta_{0}
$$
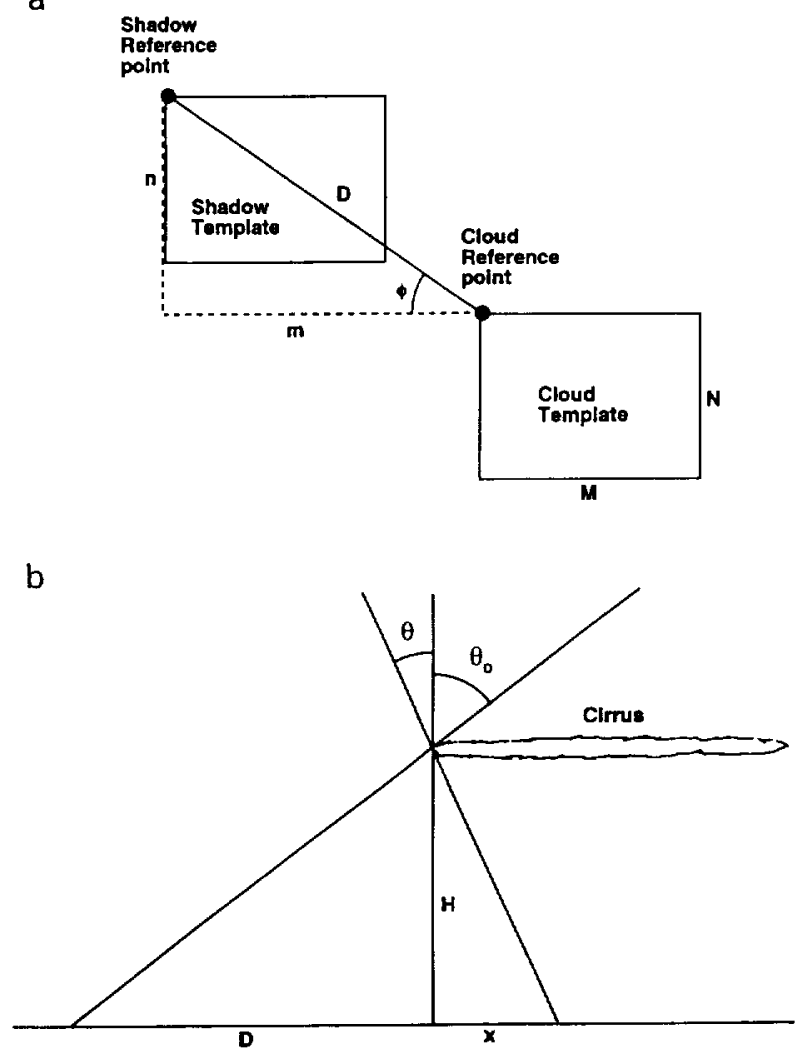

FiG. 2. (a) The cloud and shadow reference points on the $M \times N$ templates. (b) The cloud-shadow geometry used to find cloud height, where " $x$ " is the uncorrected error due to observation angle.

Finally, as mentioned in Gonzalez and Wintz (1987), correlation also can be carried out in the frequency domain. This approach requires that the cloud and shadow template regions be of the same size, with sides of length $2^{n}$, where $n$ is an integer. Then, the fast Fourier transform (FFT) can be applied. Gonzalez and Wintz (1987) report that the result often is a more accurate implementation of correlation than in the spatial domain.

The previous discussion assumes that the satellite observes the cloud at nadir. However, substantial errors may result for nonnadir observational angles $\theta$. For the case shown in Fig. 2b, the horizontal distance between cloud and shadow would be taken as $D+X$ instead of the correct value of $D$. Following Berendes et al. (1992), the fractional error in retrieved cloud height, $\Delta H / H$, due to nonnadir observation angles is

$$
\Delta H / H=\tan \theta \cot \theta_{0},
$$

where $\Delta H$ is the absolute error in height, $H$ is height, and $\theta_{0}$ is solar zenith angle.

The swath width of Landsat imagery is $185 \mathrm{~km}$; therefore, the horizontal distance along the ground from the nadir point to the edge of the image is 92.5 
$\mathrm{km}$. For Landsats $1-3$, the orbital height is $920 \mathrm{~km}$, so that the maximum observation angle $\theta$ at the edge of the image is $\tan \theta=92.5 \mathrm{~km} / 920 \mathrm{~km}$ or $\theta=5.74^{\circ}$; for Landsats 4 and 5 , the orbital height is $705 \mathrm{~km}$, so that the maximum observation angle at the edge of the image is $\theta=7.47^{\circ}$. In this study, the zenith angle varies from $34^{\circ}$ to $59^{\circ}$ for the MSS data and $67^{\circ}$ to $78^{\circ}$ for the TM data, so that the maximum possible error in cloud height retrievals is $\Delta H / H=0.12-0.15$ for scenes $\mathrm{A}$ and $\mathrm{B} ; \Delta H / H=0.06-0.10$ for scenes $\mathrm{C}-\mathrm{H} ; \Delta H / H$ $=0.03-0.05$ for scenes $\mathrm{I}-\mathrm{L}$.

This means a bias of up to $15 \%$ in cloud height may occur in the worst case scenario if the off-nadir effect is not taken into account. The cloud-to-shadow distance (see Fig. 2b) on the east side of the swath will be too large, leading to overestimates of cloud height, while on the west side of the swath this error leads to underestimates of cloud height. Following Berendes et al. (1992), off-nadir corrections are made for cloud height.

The technique applied in this paper is similar to the Berendes et al. (1992) technique in that it estimates the displacement of a shadow from the cloud. However, it differs in two respects. First, it estimates the displacement of a shadow segment or subregion from the corresponding cloud segment or subregion that casts the shadow. That is, it estimates the displacement in an average or local sense in which every pixel in the cloud and shadow subregions contribute to the result. This is in contrast to the Berendes et al. (1992) technique that finds the displacement between specific points on the shadow and cloud edges. Second, in the Berendes technique, shadow and cloud edge points were specifically selected to retrieve cloud-base height of optically and physically thick clouds. In this technique, the height retrieved is a function of the shadow and cloud subregions selected for cross correlation. In addition, the clouds analyzed in this study are optically and physically thinner and, within the certainty interval of the height retrieved for a given cloud, the notion of cloud top or base may be meaningless.

\section{a. Limitations}

There are a number of limitations of this method. First, as in the Berendes technique, it is applicable only during the daytime since it is based on near-IR channels. Second (again, as in the Berendes technique), if the cloud shadow falls upon a sloping surface, the cloud will be retrieved with one side higher than the other, also leading to errors in retrieved cloud height. However, the ability to correct for this type of error requires high-spatial-resolution digital elevation models (DEMs). No attempt is made in this preliminary investigation to correct for surface slope effects. Lastly, the method is limited to clouds of moderate to large optical thicknesses that cast discernible shadows. Also, note that the cloud template must be chosen so that the shadow template can be located on a surface without interference from nearby clouds.

\section{b. Interpretation of the retrieved cloud height}

To demonstrate the meaning of the retrieved height from this technique with respect to cloud top or base, consider the illustration in Fig. 3. The point labeled CS in Fig. 3 indicates a region on the sunside of the cloud, while the point labeled SS indicates the corresponding shadow region. Likewise, the point labeled CA indicates a region on the antisunside of the cloud, while the point labeled SA indicates the corresponding shadow region.

The cloud-shadow geometry represented by CS and $\mathrm{SS}$ is the most desirable for determining the cloud-base height. When cross correlating these two regions, the distance labeled as DS is retrieved. The error associated with this type of retrieval [and is also common to the Berendes et al. (1992) technique] is labeled as $\epsilon_{1}$ and is due to the displacement between that part of the cloud depicted in the imagery and the part of the cloud casting the shadow. This error increases with increasing solar zenith angle because the area of the cloud casting the shadow moves farther away from the sunside edge of the cloud. In this case, the tendency is to overestimate cloud-base height. If we assume that the cloud base is relatively flat, this error is small. A mitigating factor, also due to the illumination geometry, is the small incremental height per pixel for large solar zenith angles. For example, when the solar zenith angle is $75^{\circ}$, each pixel corresponds to $6 \mathrm{~m}$ of height change (assuming a spatial resolution of $28.5 \mathrm{~m}$ ), so that errors of a few pixels result in only $20-30 \mathrm{~m}$ of error in retrieved cloud-base height.

Many times the only apparent corresponding cloud and shadow features in satellite imagery are located on

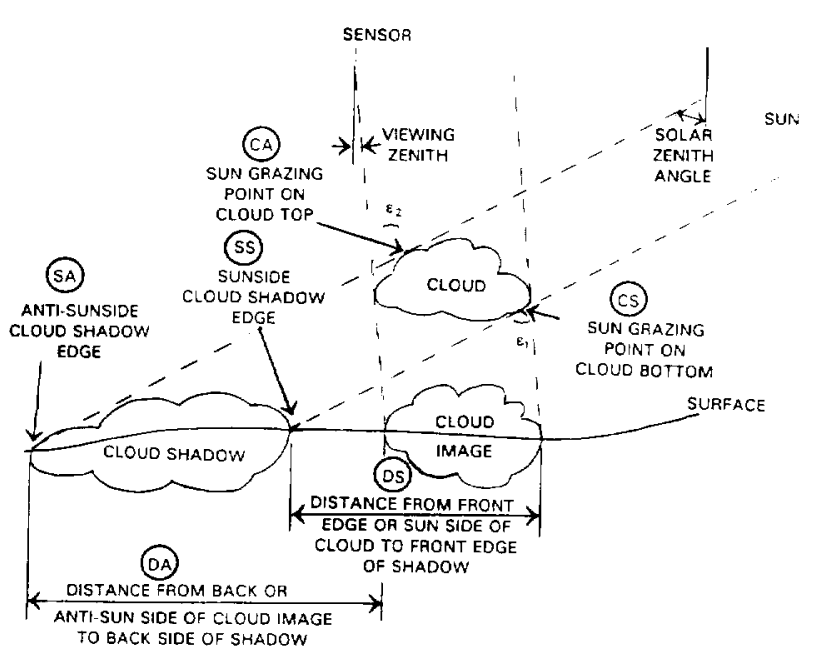

FIG. 3. Illustration of the matching cloud-shadow pair geometry. The annotations are described in the text. 
the antisunside of the cloud. Regions like this are labeled as CA and SA, respectively, in Fig. 3. In this case, the retrieved cloud height corresponds more closely to an upper cloud level (higher than the base), depending on the cloud shape and solar zenith angle. Here the interpretation of the retrieved cloud height is more subjective than when retrieving cloud-base height. If the solar zenith angle is small, then potentially a level close to cloud base is being retrieved. If the solar zenith angle is large and the cloud top is relatively flat, then the retrieved height corresponds closely to cloud top. If the solar zenith angle is in the range of $30^{\circ}-60^{\circ}$, then, depending on the cloud structure, the retrieved height corresponds to some middle cloud level. Here again an error is introduced due to the solar-illumination geometry. The portion of the cloud on the back or antisunside that casts the shadow usually is not at the cloud edge ( see $\epsilon_{2}$ in Fig. 3). This results in an underestimation of the apparent cloud-top height. Unlike the case described previously for the sunside of the cloud, the errors here are potentially larger, since the shape of the top of the cloud may not be flat. The cloud shape may be approximated using the Landsat infrared channel (Kuo et al. 1993). Then a much more detailed analysis of cloud-height variations can be made. While beyond the scope of this concept-based paper, this approach will be applied in a follow-up investigation.

\section{Results}

The 13 scenes used in this investigation are shown in Figs. 1 and 9. Subregions of $512 \times 512$ pixels from each of the scenes in Fig. 1 are shown in Fig. 4 and a subregion of $750 \times 750$ pixels is shown in Fig. 9. In the present investigation, these subregions have been selected manually; however, a fully automated classification scheme could be used to identify cirrus and stratus clouds (e.g., Kuo et al. 1988; Welch et al. 1989; Chen et al. 1989; Lee et al. 1990). The smaller regions shown as boxes in Fig. 4 are used in the following analysis. The analysis of scene $M$ (Fig. 9) is discussed in section $4 d$.

\section{a. Retrieval of cloud height using cross correlation}

Figure 5 a shows a $512 \times 512$ pixel subregion over China (scene A ). A smaller $128 \times 128$ pixel region of cirrus clouds is indicated by the white box in the center of the image. The corresponding shadow box to the upper left is the region that has the highest correlation, as given by (1). An expanded view of the selected cirrus subregion is shown in Fig. 5b, and the FFT for this region is shown in Fig. 5c. The inverted image of Fig. $5 \mathrm{~b}$ is shown in Fig. 5d, and its corresponding FFT is shown in Fig. 5e. The best-fit shadow region is shown in Fig. 5f and its FFT is shown in Fig. $5 \mathrm{~g}$. In this procedure, the shadow regions of size $128 \times 128$ pixels are correlated with the inverted cloud region using FFTs. The shadow region with the largest correlation coefficient is selected. In this particular case, the best-fit correlation coefficient is 0.93151 . The retrieved height is estimated to be $9997 \mathrm{~m}$.

Figure 6 a shows a similar $512 \times 512$ pixel subregion of scene F. A smaller $64 \times 64$ pixel region of cirrus clouds is indicated by the white box in the lower portion of the image. The best-fit correlation coefficient is 0.89404 in this case, and retrieved height is estimated as $9293 \mathrm{~m}$. A similar image is shown in Fig. 6b (scene $H$ ) for a subregion of size $64 \times 128$ pixels. The cloud region (highlighted by a black box) is to the far right side of the image, and the corresponding shadow region (highlighted by a white box) is to the upper portion of the image. The best-fit correlation coefficient is 0.62090 in this case, and retrieved height is estimated as $7597 \mathrm{~m}$. Finally, a low stratocumulus cloud deck off the coast of Antarctica is shown in Fig. 6c (scene I). In this case, the subregion selected is $256 \times 128$ pixels in size. The cloud region is shown in the center-right of the image, and the shadow region is to the lower left (both highlighted by white boxes). Note the presence of broken sea ice in the shadow region. The best-fit correlation is 0.83157 in this case, with an estimated retrieved height of $2388 \mathrm{~m}$. The presence of broken sea ice does not significantly impair the retrievals.

In all four of these height retrievals (i.e., Figs. 5 and $6), 1)$ the cross correlated shadow-cloud region pairs correspond to the antisunside and 2) the solar zenith angles are greater than $40^{\circ}$; therefore the retrieved height probably corresponds to a middle to upper level of the cloud (i.e., Fig. 3 ). In the case of scene I, because of the very large solar zenith angle (i.e., $67^{\circ}$ ), the retrieved height probably corresponds more closely to the top.

The retrieved values of cloud height and best-fit correlation coefficients are shown in Table 2 for the 12 subregions of Fig. 1 selected in this investigation. Cloud heights are found to vary from about $1106 \mathrm{~m}$ in case $L$ to $11307 \mathrm{~m}$ in case $C$. Correlation coefficients are found to vary from a high of about 0.93 in scene $A$ to a low of about 0.26 for scene $G$. Our interpretation of the retrieved height (i.e., top, base) also is indicated.

\section{b. Verification of retrieved height}

To demonstrate the accuracy of this technique for estimating the displacement between a selected cloud edge and its corresponding shadow edge, a manual process is used. Through visual examination of the imagery, salient features in the cloud structure can be identified along with their corresponding shadow feature on the surface. The distance between these features then can be compared with that derived from the $2 \mathrm{D}$ cross correlation technique of the previous section.

Figures $7 \mathrm{a}-\mathrm{d}$ shows small subregions extracted from each of the four Antarctica scenes (i.e., scenes I, J, K, 

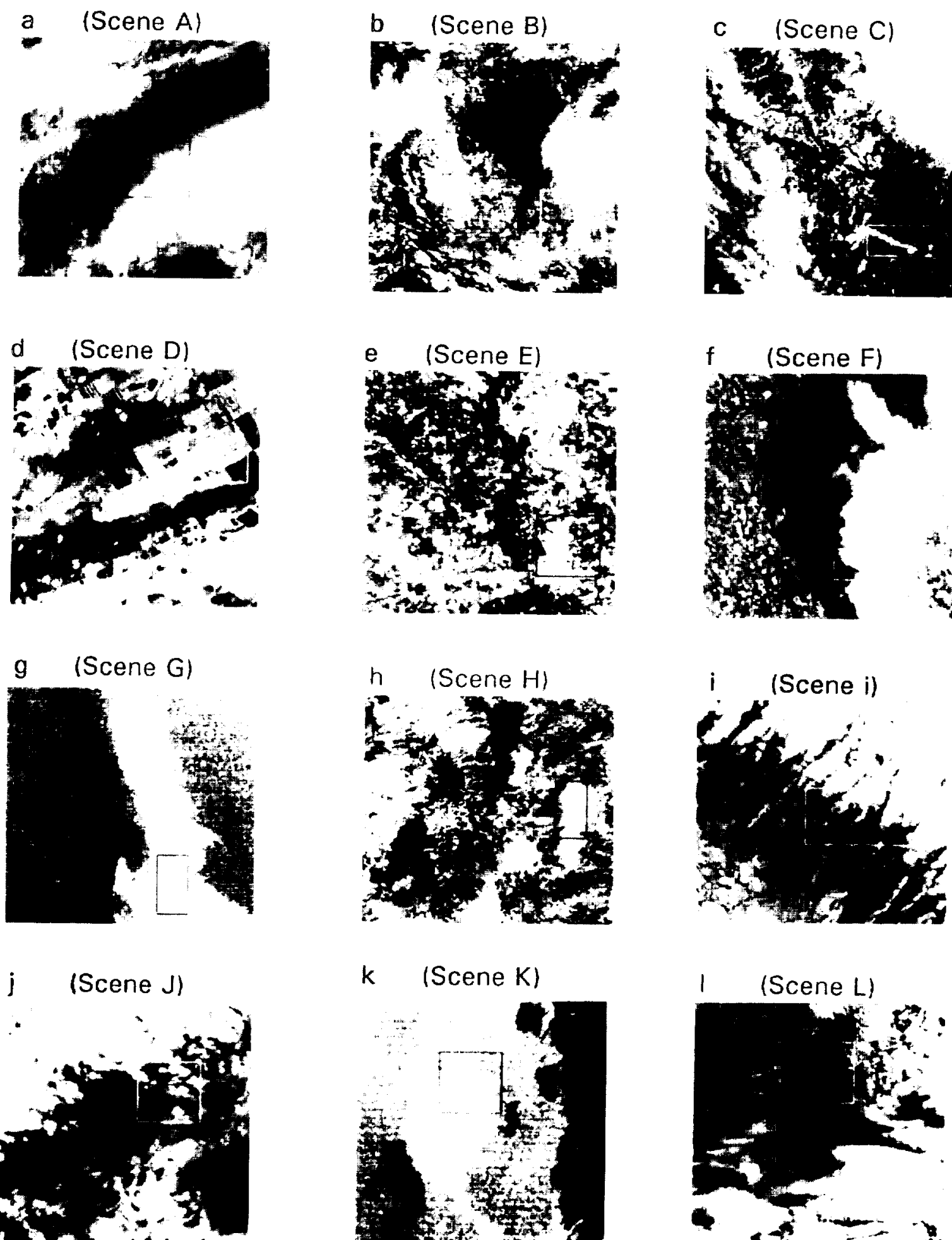

k (Scene K)

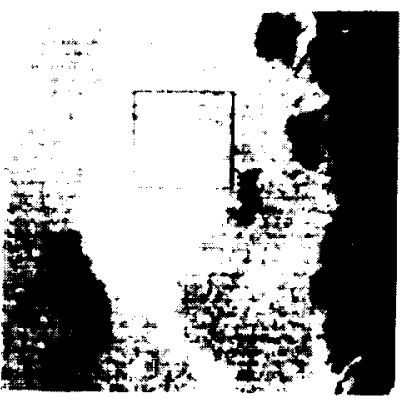

1 (Scene L)

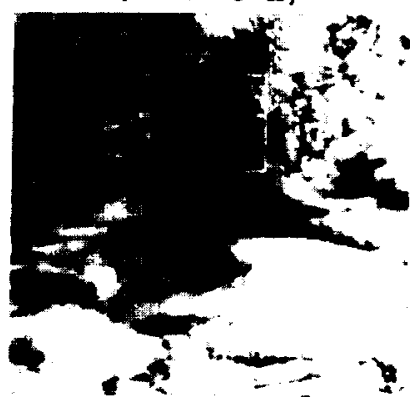

FIG. 4. (a)-(1) $512 \times 512$ subregions used in the analysis from Fig. 1 . 
a

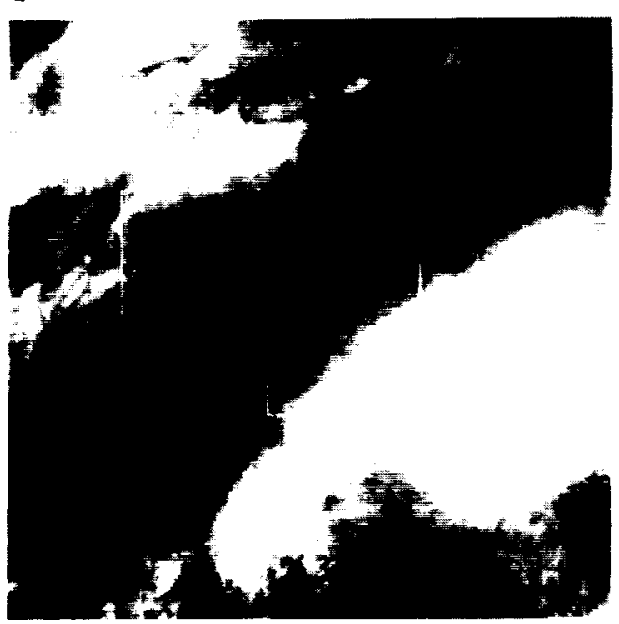

b

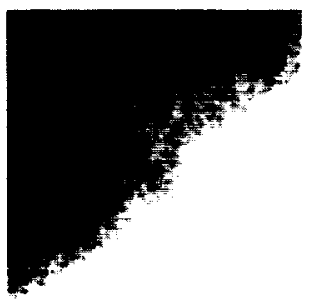

d

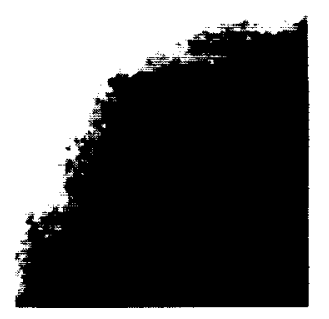

$f$

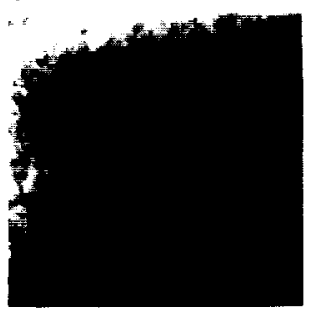

Fic. 5. (a) $512 \times 512$ subregion of scene A; (b) $128 \times 128$ pixel cloud subregion (center box); (c) FFT of (b); (d) inverted image of (b): (c) FFT of (d); (f) shadow region (box in upper left); (g) FFT of (f).

and $L$ ) that contain cloud and shadow features that can be used in a manual cloud-shadow matching technique. In each subregion a ruler is superimposed on the imagery to indicate distance in height above the surface for any point of interest that coincides with a point on the ruler. The tic marks and numbers on the ruler indicate kilometers above the surface. The origin of the ruler is placed at the location of a salient cloud feature, and the location of the appropriate shadow feature on the ruler gives the height of the cloud. One large vertical mark is indicated on the ruler corresponding to the cloud height determined from the cross correlation method. Note that the scenes have been rotated such that the solar azimuth is aligned along the horizontal direction and the solar illumination is originating from the left side of the figure. Upon examination of Figs. $7 a-d$, it can be seen that the cloud height retrieved from the cross correlation method coincides closely to the value one would select manually, well within plus or minus $10 \%$ of the total cloud height.

In Figs. $7 a$ and $7 b$ the cross correlated cloudshadow pairs correspond to the antisunside. The large solar zenith angles in these two scenes suggest that the retrieved height is close to cloud top. In Figs. $7 \mathrm{c}$ and $7 \mathrm{~d}$ both sunside and antisunside cloud-shadow pairs are apparent. The rulers are placed at sunside cloudshadow pair locations and the retrieved height is indicative of cloud-base height. Some additional cloudshadow pairs are manually selected in these two figures and are annotated as " $O$ " and " $x$ " for the sunside and antisunside, respectively. The cloud-base height retrieved from the " $\bigcirc$ " pairs is indicated to the right as " $H=$." To demonstrate the potential for using cloudshadow pairs from both the sunside and antisunside for retrieving cloud thickness, the retrieved height from the corresponding " $x$ " pairs is differenced with the cloudbase height and is also shown to the right as cloud thickness (i.e., " $T=$ "). Note that in both figures the retrieved cloud-base heights from the manually selected points agree with those values retrieved from the cross correlation method within plus or minus $20 \mathrm{~m}$. The retrieved values for cloud thickness show larger variability but suggest that the cloud in Fig. 7c is between 85 and $125 \mathrm{~m}$ thick, while that in Fig. $7 \mathrm{~d}$ is on the order of 60-80 m thick. The appearance of these clouds suggests that these retrieved thickness values are reasonable.

\section{c. Comparison of retrieved heights with a temperature-based height-retrieval method}

As a means to validate the heights retrieved from the cross correlation method, cloud-top height is retrieved using cloud-top temperature. If the temperature structure of the atmosphere as a function of height is known from a sounding, then cloud-top height can be inferred. Of course, in the MSS scenes selected for analysis in this study, a thermal IR channel is not available for a temperature-based retrieval, which only leaves the shadow based approach. For the four Landsat TM scenes of Antarctica (i.e., scenes I, J, K, and L) selected for this study, a thermal IR channel is available; how- 

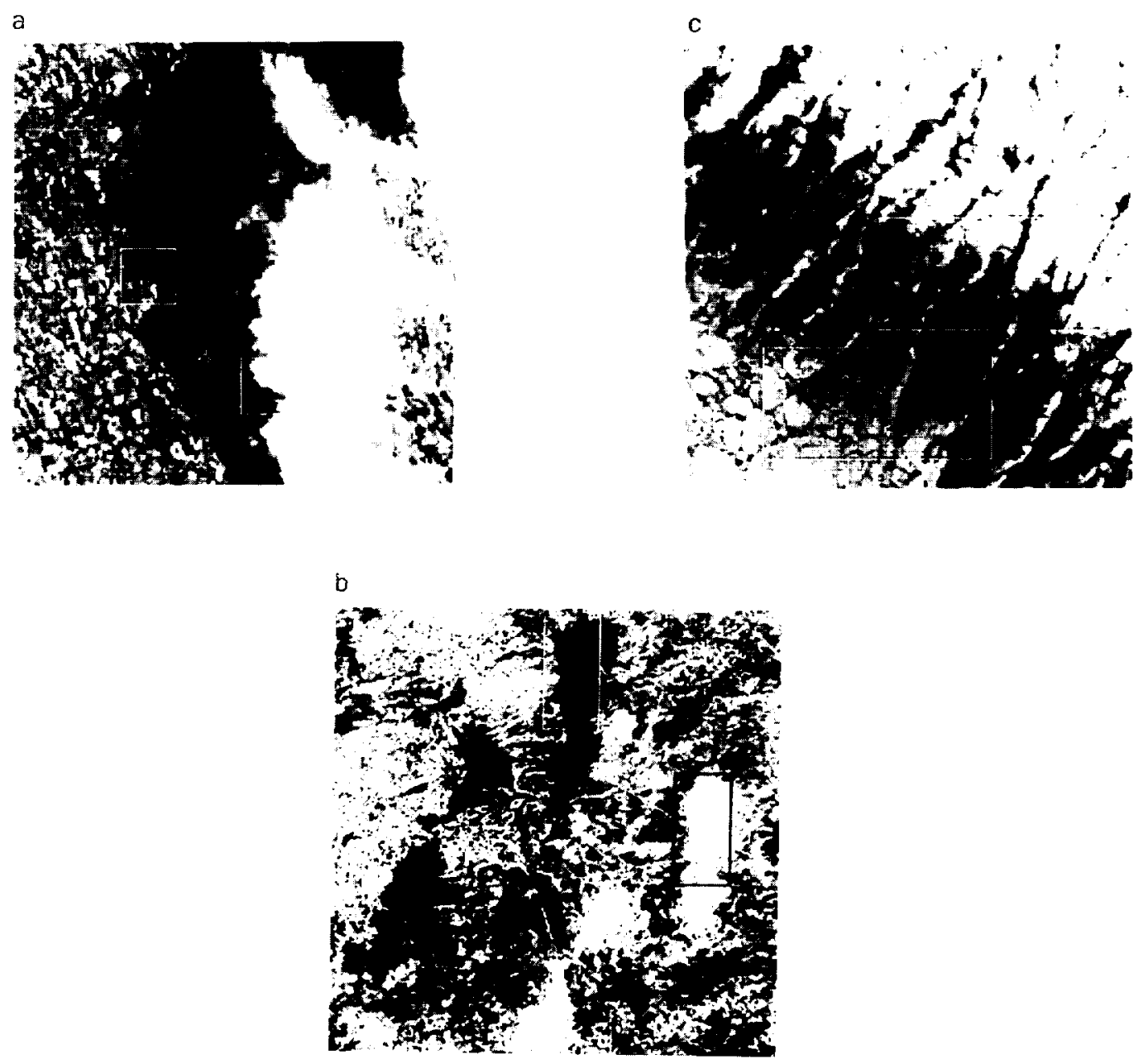

FIG. 6. (a) Same as Fig. 5a but for scene F using a $64 \times 64$ pixel subregion. (b) Same as Fig. 5a but for scene H using a $64 \times 128$ pixel subregion. (c) Same as Fig. 5a but for scene I using a $256 \times 128$ pixel subregion.

ever, soundings from Antarctica are sparse, to say the least. As a surrogate for a sounding, a vertical temperature structure for each of the Antarctica scenes (i.e., scenes $\mathbf{I}, J, K$, and $L$ ) was extracted from the mean monthly European Centre for Medium-Range Weather Forecasts (ECMWF) model analyses for the $2.5^{\circ}$ grid closest to the location of scene center. The ECMWF analyses were obtained from the National Climatic Data Center (NCDC) CDROM of Global Upper Air Climatic Atlas (GUACA). In Figs. 8a-d the mean vertical temperature profiles for the month for each of the scenes is shown in addition to plus and minus one standard deviation. The cloud height determined from the cross correlation method also is indicated as a solid horizontal line. The error bounds on the retrieved cloud height for these scenes are estimated to be $50 \mathrm{~m}$, based on examination of the rulers in Fig. 7, and are potentially larger due to the $\epsilon_{2}$ error described in section 3 and depicted in Fig. 3. The vertical dashed lines in Figs. $8 \mathrm{a}-\mathrm{d}$ show the mean cloud-top temperatures obtained from the Landsat TM data for the clouds of interest. Unfortunately, the Landsat TM thermal IR data is known to have a bias (Orheim 1988). However, through a simple vicarious method, we adjust the cloud-top temperatures in the following way. In a number of areas in this imagery, small areas of open water occur in the broken sea ice. We assume the actual temperature of the water is $271 \mathrm{~K}$ and adjust the cloud-top temperatures by the difference between the apparent temperature and $271 \mathrm{~K}$. In this imagery, the adjustment is approximately $7^{\circ}$. This unsophisticated method does not take into account the attenuation due to the atmospheric path between the surface and the cloud top, but we assume that the polar atmosphere is dry and therefore the atmospheric effects are small. In Figs. 8a, 8b, and $8 \mathrm{~d}$ the retrieved cloud height from the shadowbased method coincides with the temperature-based method within the range of plus and minus one standard deviation of the temperature profile. Note that the temperature profiles from the ECMWF analyses are refer- 
enced to sea level, and in Fig. 8d the height retrieved by the cross correlation method was adjusted by approximately $700 \mathrm{~m}$ to compensate for the height of the surface above sea level. The cloud heights for Figs. 8ac did not require any adjustment, as the surfaces in these scenes are already at sea level; that is, they are from the Weddell Sea and Prdyz Bay. In Fig. 8c there is a large disparity between the height retrieved from the cross correlation method and the cloud-top height inferred by the temperature profile. If we assume that the Landsat TM thermal IR data (i.e., Band 6) for this scene is good, the disparity can only be explained by an extraordinary variation in the temperature profile from the mean for the date of this overpass.

\section{d. A special validation case}

One additional scene (to those in Fig. 1) was analyzed and is shown in Figure 9 (i.e., scene $\mathbf{M}$ ). This scene was reserved for this validation section for two reasons. First, it contains an optically very thin cirrus cloud that is virtually transparent everywhere and requires a special procedure to retrieve cloud-top height from the temperature profile. Second, it is data that has a thermal IR channel (i.e., Landsat TM) for which radiosonde soundings are available on the day of the overpass. For this scene the 0000 and 1200 UTC sounding bracketing the overpass time provides for a direct comparison between the temperature and cross correlation retrieved heights.

It is difficult to retrieve the cloud-top height from the temperature-based method since this cloud has an emissivity of less than 1. Cloud-top height cannot be retrieved without first correcting the apparent cloud-top temperature for emissivity. A technique for correcting the temperature is outlined in Minnis et al. (1993); however, knowledge of cloud optical depth is required. Optical depth can be retrieved with some assumptions about the cloud particle (i.e., ice crystal) distribution, type (i.e., plates, columns, etc.), and background al-

TABIE 2. Retrieved cloud heights, correlation coefficients, and azimuth errors as a function of scene.

\begin{tabular}{lcccl}
\hline Scene & $\begin{array}{c}\text { Correlation } \\
\text { coefficient }\end{array}$ & $\begin{array}{c}\text { Height } \\
(\mathrm{m})\end{array}$ & $\begin{array}{c}\text { Azimuth } \\
\text { error }\end{array}$ & $\begin{array}{c}\text { Height } \\
\text { retrieved }\end{array}$ \\
\hline A & 0.932 & 9997 & -0.5 & top \\
B & 0.547 & 9998 & -1.3 & base \\
C & 0.425 & 11307 & -1.2 & base \\
D & 0.765 & 6432 & -0.3 & top \\
E & 0.414 & 7166 & 0.1 & base \\
F & 0.894 & 9293 & -1.5 & top \\
G & 0.263 & 10216 & 1.4 & top \\
H & 0.621 & 7597 & 1.5 & top \\
I & 0.832 & 2388 & 0.3 & top \\
J & 0.506 & 2136 & 0.5 & base \\
K & 0.478 & 1300 & 0.1 & base \\
L & 0.805 & 1106 & 0.2 & top \\
\hline
\end{tabular}

(a) Scene I

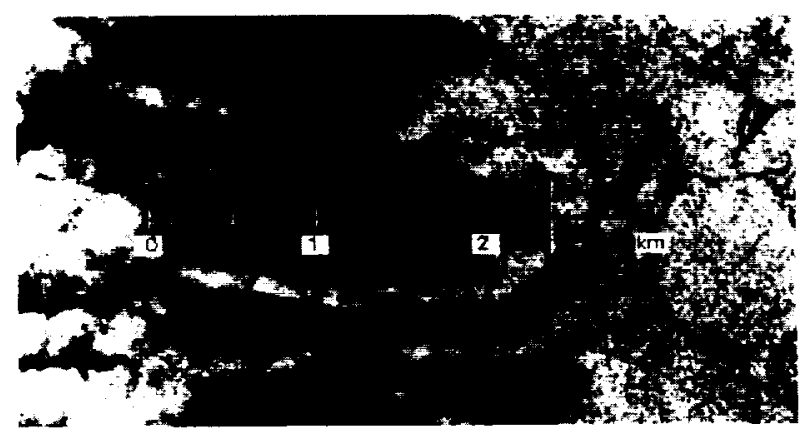

(b) Scene L

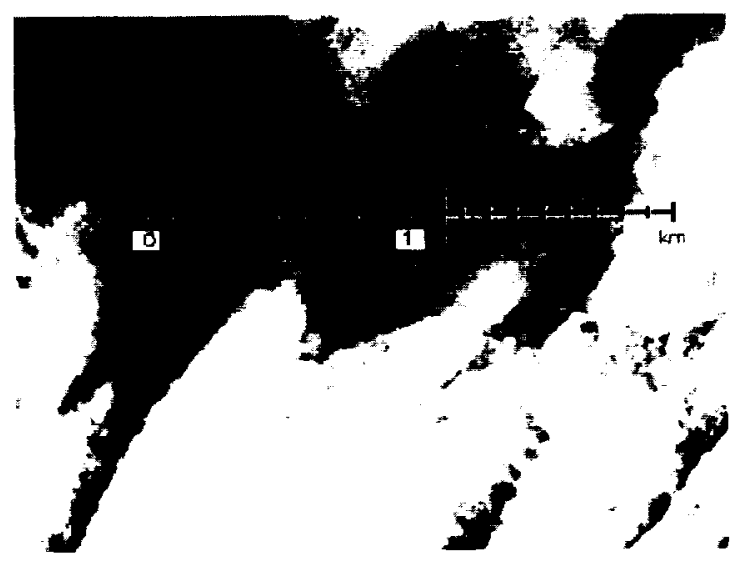

Fic. 7. Manual verification of retrieved cloud heights from cross correlation method. Rulers indicate height in $\mathrm{km}$ from the origin. Here (a) and (b) are extracted from scenes I and $L ;$ (c) and (d) are extracted from scenes $J$ and $K$. The " $O$ " and " $x$ " annotations in (c) and (d) are described in the text.

bedo (e.g., King et al. 1992); however, there is some uncertainty in the retrieved optical depth value

It is difficult to retrieve the cloud height from the cross correlation method due to the indistinct edges of the cloud and shadow, and in this case the cloud is virtually transparent everywhere. One advantage of using the cross correlation method is that it does not necessarily require distinct cloud and/or shadow edges for it to be applicable. The factor that confounds the cross correlation the greatest is the high brightness variability of the background. For example, the surface depicted in Fig. 9 is dominated by a patchwork of agricultural land, and the surface brightness can vary from very dark to very bright depending on whether the ground has been tilled or not. The nonuniform background areas in and around the cloud and shadow areas that are cross correlated can skew the cross correlation function away from the desired location. The effect of 
(c) Scene J

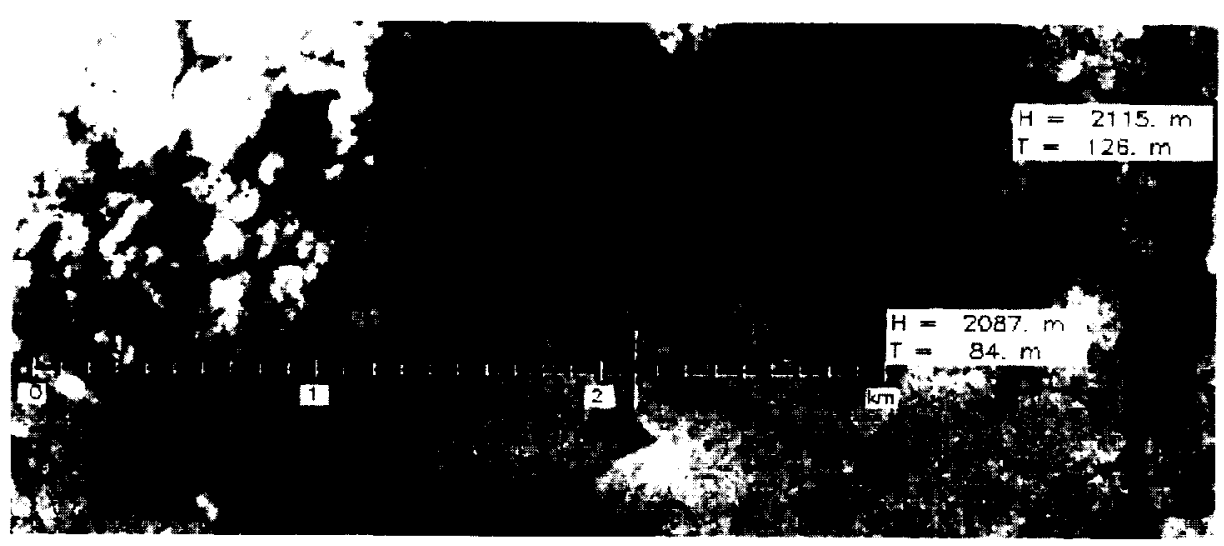

(d) Scene K

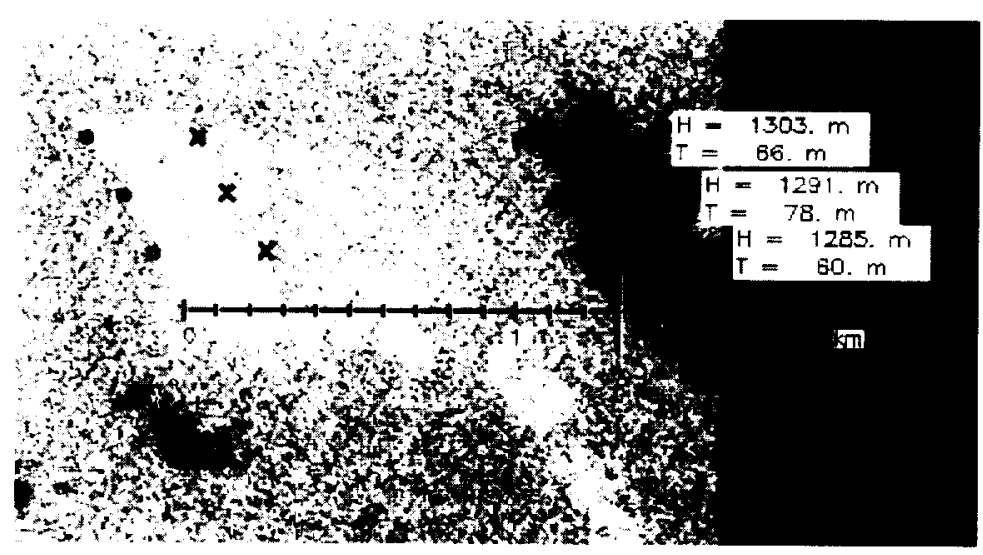

FiG. 7. (Continued)

the background on the cross correlation method in this imagery can be mitigated somewhat by using the gray flip of the thermal IR channel of Landsat TM (i.e., band 6 ) when selecting the cloud region. (Note that the band 6 imagery is not shown.) In band 6 the background appears homogeneous and the cold, cirrus cloud contrasts well with the background. The shadow region to cross correlate with the cloud region is then selected from band $5(1.55-1.75 \mu \mathrm{m})$ which was found from experience to provide the best contrast between shadowed and unshadowed areas in this scene. Note that in other scenes this may not necessarily be true.

In Fig. 9 the imagery is rotated as in Fig. 7 such that solar azimuth is aligned with the horizontal. Once again, a ruler is superimposed on the imagery to give a sense of the approximate height of the cloud. It is much more difficult to gauge the cloud height in this scene than for those in Fig. 7, but it appears that the value of approximately $12 \mathrm{~km}$ retrieved from the cross correlation technique is accurate to within plus or mi- nus $1 \mathrm{~km}$. Because the solar zenith angle is much smaller than in the Antarctica scenes and since the cloud and shadow edges are from the back or antisunside of the cloud, we are probably retrieving a cloud height from the middle of the cloud. However, because this cloud is not only optically thin but also physically thin (probably less than $1 \mathrm{~km}$ ), and since the accuracy is not less than $1 \mathrm{~km}$, there is no point in determining whether the retrieved $12-\mathrm{km}$ height corresponds to the base or the top of the cloud.

Figure 10a shows the soundings before and after the time of the overpass (i.e., 1200 UTC 14 June 1984 and 0000 UTC 15 June 1984) from Longview, Texas. There is little difference between the two soundings over the 12-h period, and we can probably assume that there is not much difference at the time of the overpass. The retrieved cloud height from the shadow-based method is indicated as a solid horizontal line with plus and minus $1-\mathrm{km}$ error bounds indicated. In Fig. 10b, surface temperature is plotted as a function of corrected 

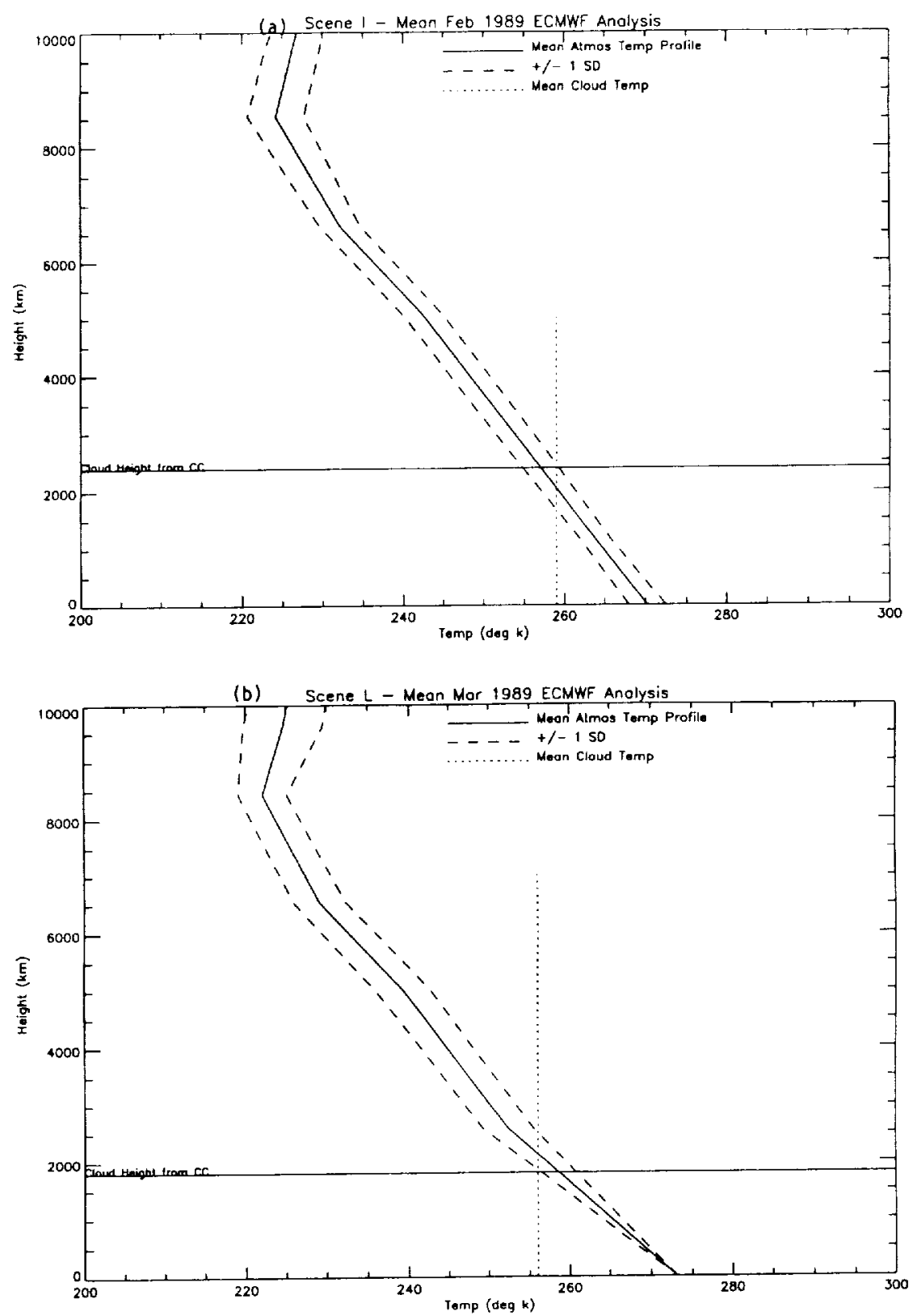

FiG. 8. Temperature profiles derived from ECMWF analyses for scenes (a) I, (b) L, (c), J, and (d) K.

cloud-top temperature for a given 238-K apparent cloud-top temperature for six different values of optical depth. The curves are derived using the technique of Minnis et al. 1993. The apparent cloud-top temperature of $238 \mathrm{~K}$ is the minimum value found for the cloud examined and probably corresponds to the optically thickest and highest part of the cloud. The mean surface temperature for the clear areas surrounding the cloud is found to be $300 \mathrm{~K}$ and is indicated in Fig. $10 \mathrm{~b}$ by a solid horizontal line. We can see from this plot that, for the two techniques to be in agreement, the optical depth of the thickest part of the cloud would have to be from 3.5 to 4.5 , which is reasonable. To retrieve the optical thickness of this cloud would require a ray-tracing model to generate the phase function for the assumed particle distribution within this cloud and a radiative transfer model. However, because the background is not homogeneous (an assumption that is required for the radiative transfer model) and it is difficult to determine the exact surface reflectance under the pixel for 

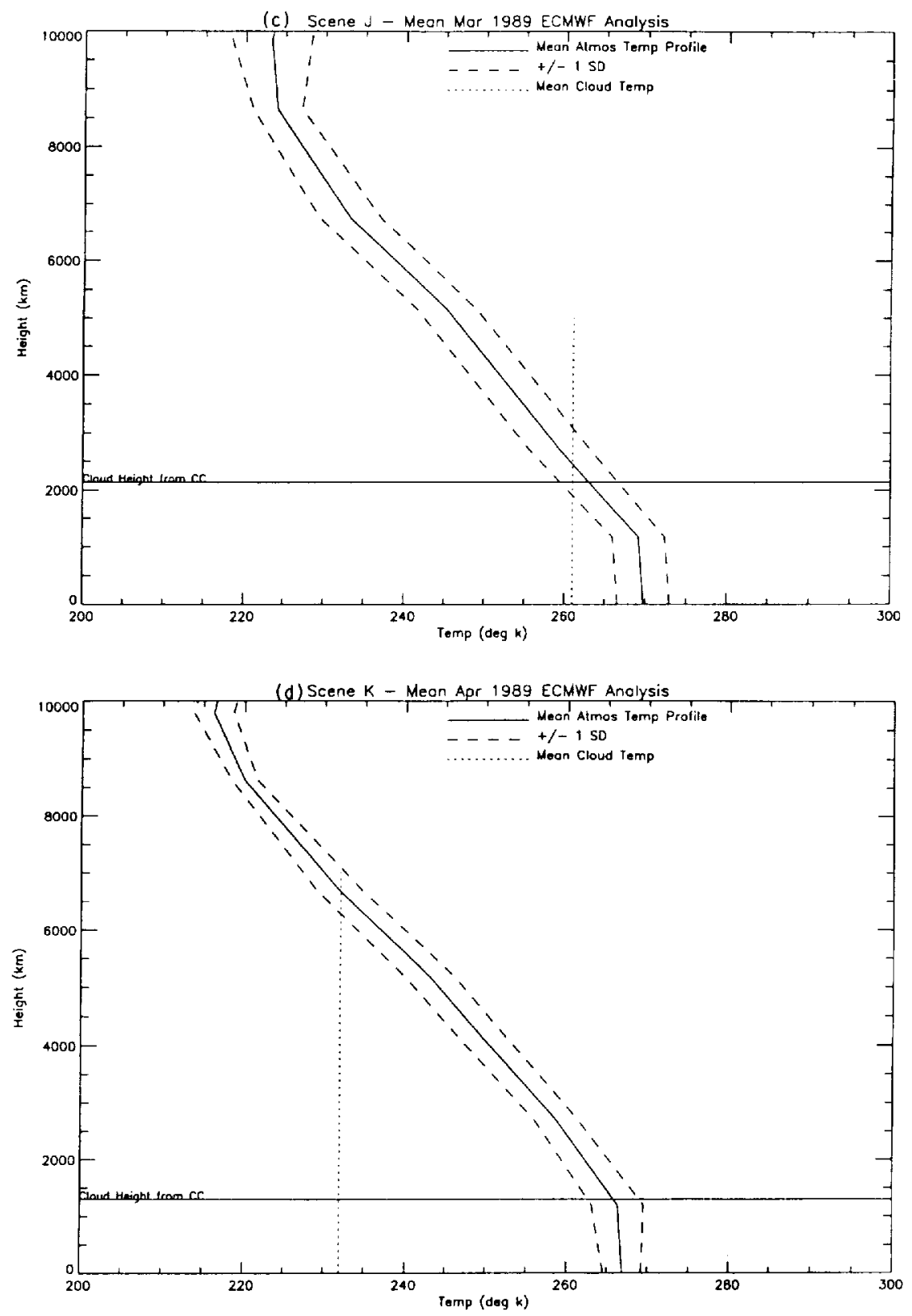

FIG, 8. (Continued)

which we are retrieving optical depth, a sufficiently accurate estimate of optical depth could not be obtained. We conclude that the temperature based method for retrieving the height of this cloud (the top or the base) is no more accurate than the shadow-based method and requires significantly more resources to perform.

\section{$e$. An indirect verification of the cross-correlation method}

Direct measurements of cloud height for the cases investigated are not available. Therefore, no exact val- idation of the results is possible. Therefore, an indirect method is employed. Both solar elevation and solar azimuth angles are available from the Landsat header files. Next, the skew angle, which is a function of latitude $L$ is computed (Mather 1987)

$$
\phi_{s}=90-\cos ^{-1}\left[\frac{\sin \left(\theta_{q}\right)}{\cos (L)}\right] \quad \text { (in degrees), }
$$

where the satellite heading (across-scan direction) at the equator is $\theta_{4}=9.09^{\circ}$ and $8.2^{\circ}$ for Landsats $1-3$ and 4 and 5 , respectively. Table 1 lists the solar zenith and 


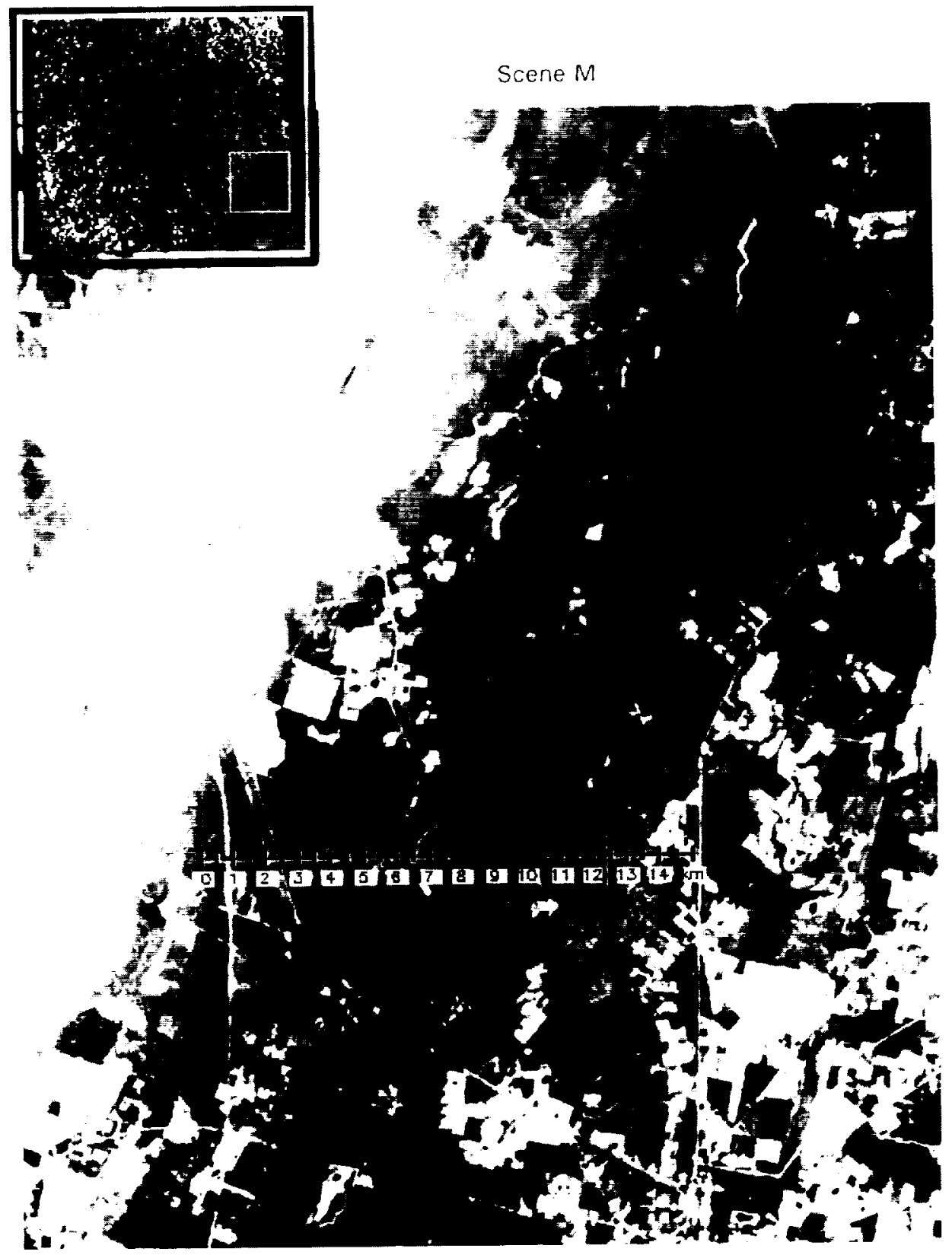

Fici 9. The $750 \times 750$ pixel subsection of Landsat TM quad scene (scene M) obtained over eastern Texas. Full quad scene is shown subsampled at upper left. The extracted subsection is highlighted in a white box. Ruler indicates height in kilometers from origin.

azimuth angles and the skew angle for the 12 scenes investigated. Then the skew angle $\phi_{s}$ is subtracted from the solar azimuthal angle $\phi_{10}$. This angle $\phi^{\prime}=\phi_{0}-\phi_{s}$ then is compared to the effective azimuthal angle computed between the cloud and shadow, $\phi$ (Fig. 2a). The difference between these two angles, $\Delta \phi=\phi^{\prime}-\phi$, is listed as "azimuth error," $\epsilon$, in Table 2. The largest azimuth error is $\pm 1.5^{\circ}$, and the average azimuth error is about $0.7^{\circ}$ for the 12 cases. These small $\epsilon$ values provide another indication that the cross correlation method accurately determines the displacement between a cloud-shadow pair.

Another test is made to determine the effect of a single pixel error in the location of the shadow region. Figure 11 shows the situation for scene A (Fig. 1). The effective azimuthal angle is $\phi=25.3^{\circ}$, and the displacements in the $x$ and $y$ directions are 133 and 63 pixels, respectively. For the eight nearest neighbor pix- 
a)

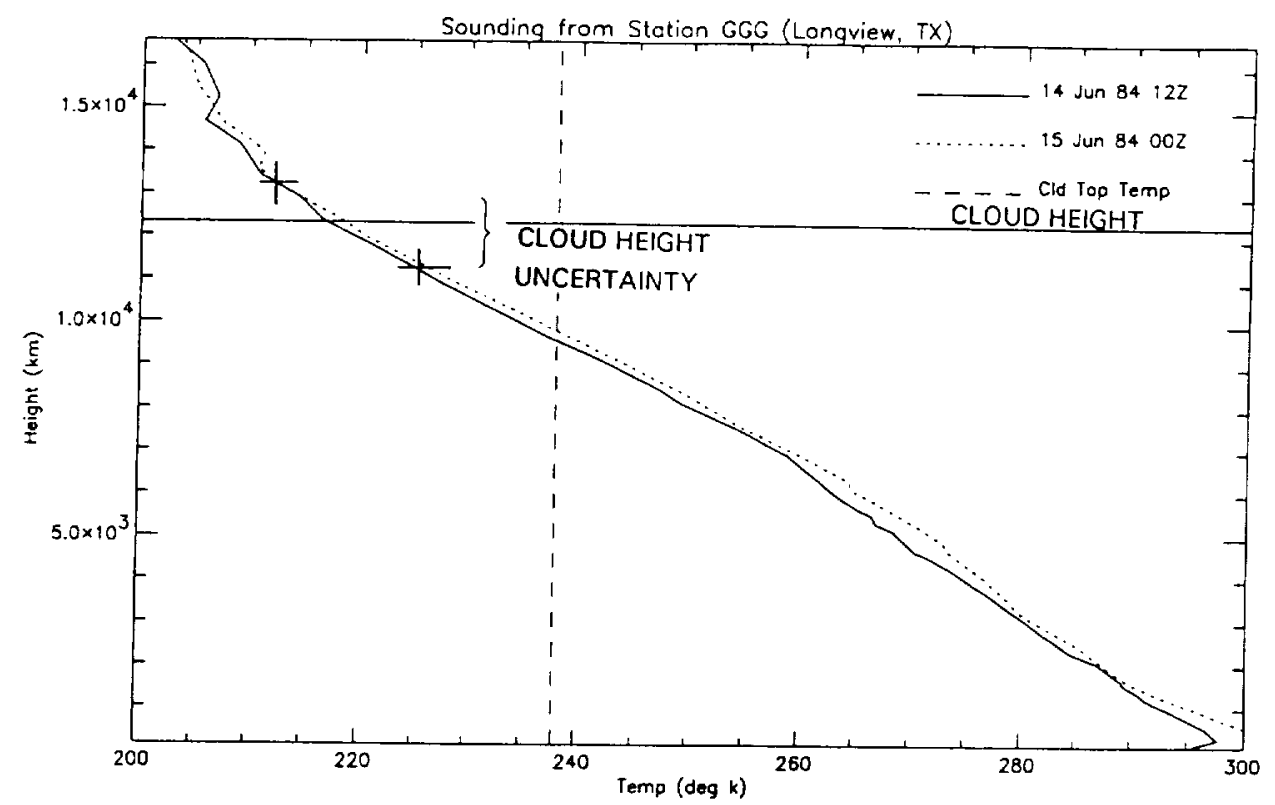

b)

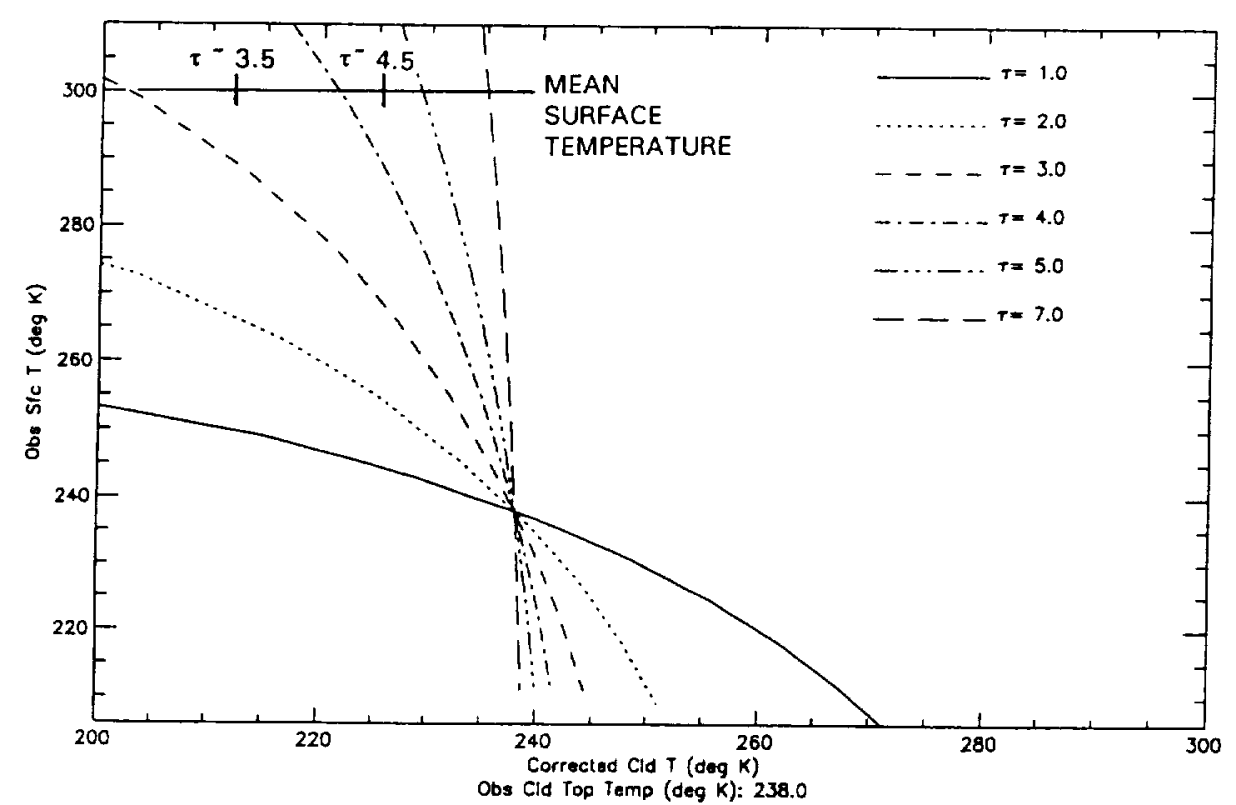

FIG. 10. (a) Radiosonde soundings bracketing time of overpass for scene $M$ in Fig. 9, (b) corrected cloud temperature versus observed surface temperature for optical depths of $1,2,3,4,5$, and 7 .

els labeled in Fig. 11, Table 3 shows the variations in $\Delta \phi$ and the azimuth error $\epsilon$ that would have resulted. In this case, the point of highest correlation (i.e., the center pixel ) yields a value of $\epsilon=-0.5^{\circ}$, whereas the pixels labeled as 2 or 3 in Fig. 6 would have yielded a smaller value of $\epsilon= \pm 0.1^{\circ}$. This suggests that the method probably is subject to at least \pm 1 pixel errors in horizontal displacement. Note, however, that the solar elevation and azimuthal angles are given as integers on the Landsat header files. Therefore, both of these angles are accurate to no better than to $0.5^{\circ}$. Furthermore, pitch, roll, and yaw motions on the satellite plat- 


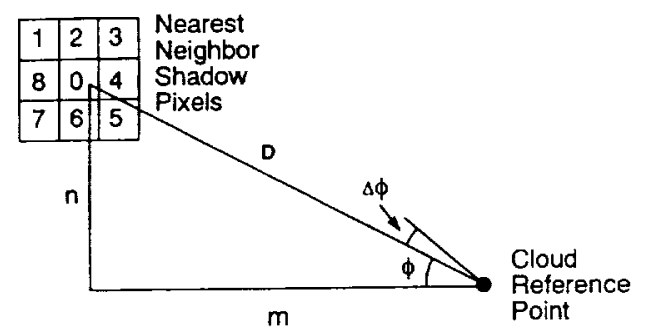

Fici. 11. The nearest neighbor array of labeled shadow pixels used for the azimuthal angle error analysis. The center pixel labeled " 0 " is the selected shadow reference point.

form introduce additional unknown variations. Therefore, it is not possible to conclude that the method is accurate in detecting horizontal displacements between cloud and shadow to better than a few pixels, corresponding to errors in cloud height of about 50 to about $250 \mathrm{~m}$.

\section{f. Sensitivity of cross-correlation method to template location}

For an automated cloud-height retrieval scheme to be developed based upon this correlation approach, both the locations of the cloud template and the size of the cloud template need to be selected by the algorithm. As mentioned previously, a robust cloud-classification scheme can be used to detect the presence of cirrus and stratus cloudiness. However, whether or not the sizes of the templates and the locations of the templates affect the results needs to be answered. Figure 12a shows four cloud subregions labeled as A, B, C, and D, each of $128 \times 128$ pixels in size for scene B (Fig. 1). The corresponding shadow regions are labeled as $\mathrm{a}, \mathrm{b}, \mathrm{c}$, and $\mathrm{d}$, respectively, in Fig. 12a. The retrieved cloud heights are $10076,10186,9998$, and $9965 \mathrm{~m}$ for subregions $a, b, c$, and d, respectively. These results suggest the retrieved cloud height is relatively constant and that the retrieval approach is robust; that is, retrieved cloud height varies by no more than about $100 \mathrm{~m}$. Figure $12 \mathrm{~b}$ shows a similar analysis, extracting four highly overlapping elongated subregions of the jet contrail from scene $C$. The retrieved cloud (contrail) heights are $11368,11212,11307$, and 11339 $m$ for the subregions labeled $a, b, c$, and $d$, respectively. The largest difference is about $150 \mathrm{~m}$. Since the contrail can be assumed to be at constant height, these results again demonstrate the robustness of the approach. Cloud subregions from scenes $F$ and $I$ are shown in Figs. $12 \mathrm{c}$ and $12 \mathrm{~d}$, respectively. The retrieved cloud heights are $9436,9254,9293$, and 9513 $\mathrm{m}$ for subregions labeled as $\mathrm{a}, \mathrm{b}, \mathrm{c}$, and $\mathrm{d}$ in scene $\mathrm{F}$, respectively, and $2431,2314,2431$, and $2423 \mathrm{~m}$ for subregions labeled as a, b, c, and $d$ in scene $I$, respectively. The largest variation in cloud height is about $250 \mathrm{~m}$ in scene F (i.e., Fig. 12c). g. Sensitivity of cross-correlation method to template size

The next question is in regard to the template sizes chosen for the analysis. Figure 13a shows highlighted white boxes of decreasing size of $128 \times 128,64 \times 64$, $32 \times 32$, and $16 \times 16$ pixel regions selected from scene B. The location of the cloud templates is labeled as A and the corresponding shadow template is labeled as a. The retrieved cloud heights are $10776,9799,9965$, and $9998 \mathrm{~m}$, respectively. The correlation coefficients decrease steadily with decreasing template size. These results suggest the use of a template size on the order of at least 64 pixels on a side. The retrieved cloud heights are $11307,11217,11184$, and $11132 \mathrm{~m}$ in Fig. 13b ( scene C); 9148, 9293, 9185, and $9148 \mathrm{~m}$ in Fig. 13c (scene F); and 2388, 2405, 2423, and $2373 \mathrm{~m}$ in Fig. $13 \mathrm{~d}$ ( scene $I$ ). In each case, $A$ is the largest and D is the smallest template size. In each case, there is a decrease in correlation coefficient with decreasing template size. It can be concluded that template sizes of about 64 or 128 pixels on a side are adequate for accurate retrievals; however, smaller template sizes on the order of 16 to 32 pixels on a side are not advisable.

TABLE 3. Errors in azimuth expressed as $\Delta \phi$ and as $\epsilon$ as a function of single pixel displacements (see Fig. 11) in the retrieval process.

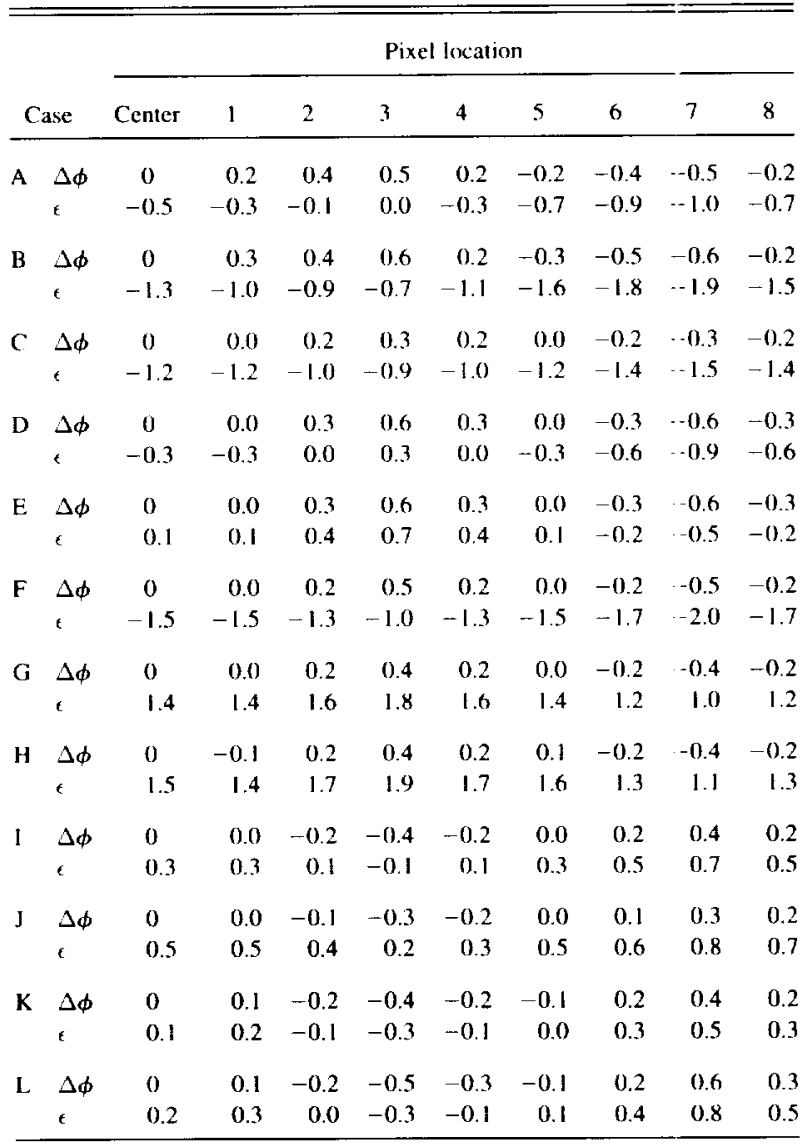



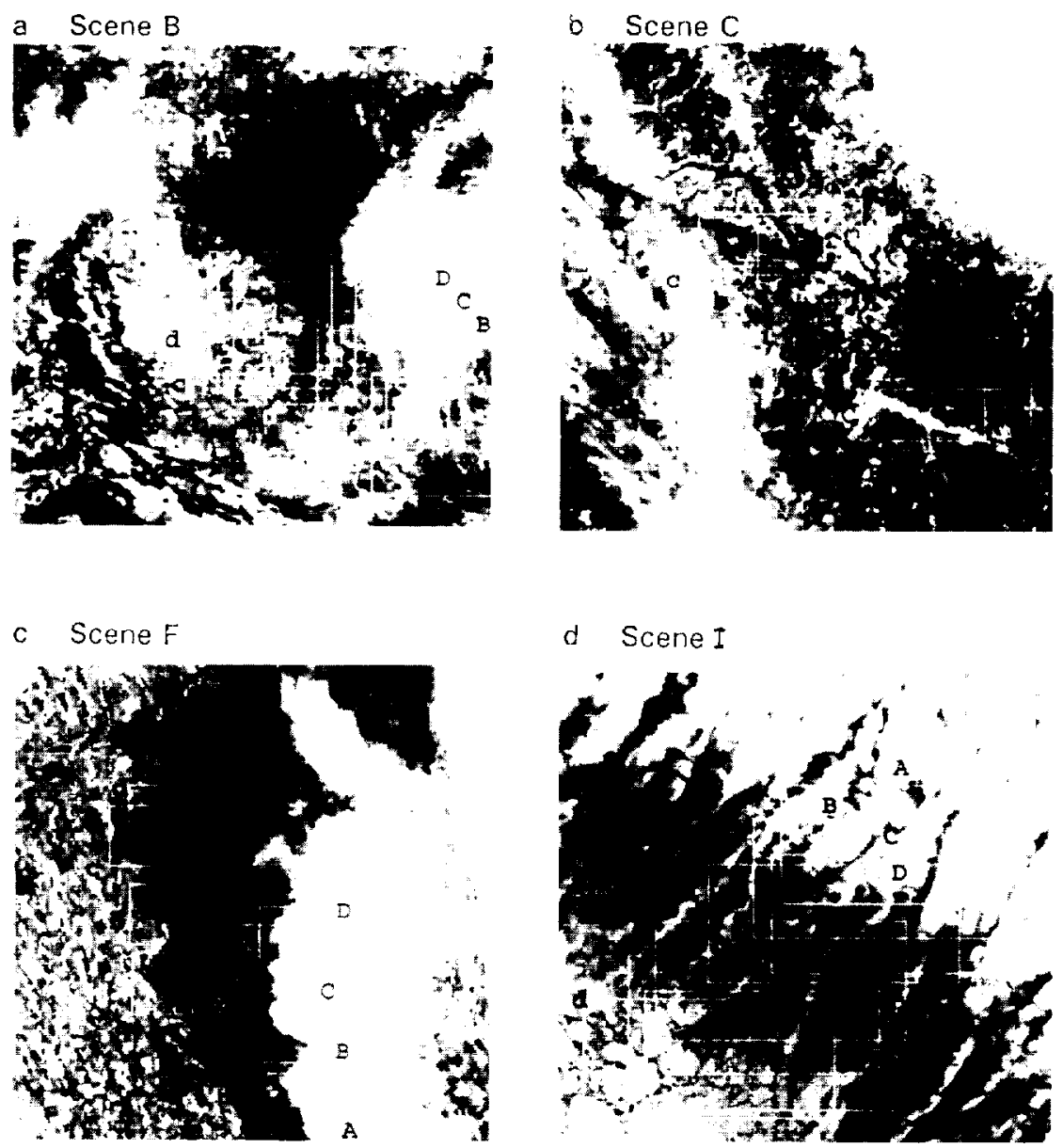

FiG. 12. Four cloud templates labeled as " $A$," "B," "C," and "D" and corresponding shadow templates labeled as "a," "b," "c," and " $d$ " for (a) scene $B$; (b) scene $C$; (c) scene F; and (d) scene I.

\section{h. Sensitivity of the cross-correlation method to sensor spatial resolution}

The final question concerns the spatial resolution necessary to apply this technique. In particular, the Moderate Resolution Imaging Spectrometer (MODIS) will be used to retrieve global cloud properties on the upcoming Earth Observing System (EOS) series of platforms. This instrument has several visible spectral channels at a spatial resolution of $250 \mathrm{~m}$. The question is whether such degraded spatial resolution is sufficient to apply this cloud-height correlation approach.

Table 4 shows the retrieved cloud heights as a function of spatial resolution for several scenes. For scene $\mathrm{E}$, the cloud-height retrieval is stable with values of about $7200 \mathrm{~m}$ up to spatial resolutions of $171 \mathrm{~m}$; then at spatial resolution of $228 \mathrm{~m}$, the retrieved height increases to about $8000 \mathrm{~m}$. Similar results are found for scene B with relatively stable retrieved values for spatial resolutions up to $171 \mathrm{~m}$ and then large increases at spatial resolution of $228 \mathrm{~m}$. These results suggest that the 250-m spatial resolution available on MODIS may not be sufficient to apply this method. However, Table 4 shows relatively stable cloud-height retrievals for the low stratus clouds over Antarctica. These stable values can be explained by the fact that these are low clouds and because the solar zenith angles are large, providing large shadow regions for the template cross correlation procedure.

\section{Conclusions}

Cloud heights typically are estimated using the 11$\mu \mathrm{m}$ infrared channel on most operational satellites; however, there is a vast array of data available from sensors like Landsat MSS that do not have thermal IR bands and do not lend themselves to a temperaturebased cloud-top height retrieval approach. Cumulus cloud-base heights have been retrieved using high-spatial-resolution imagery by matching cloud and shadow edges (Berendes et al. 1992). The purpose of the present investigation is to extend this cloud-shadow 

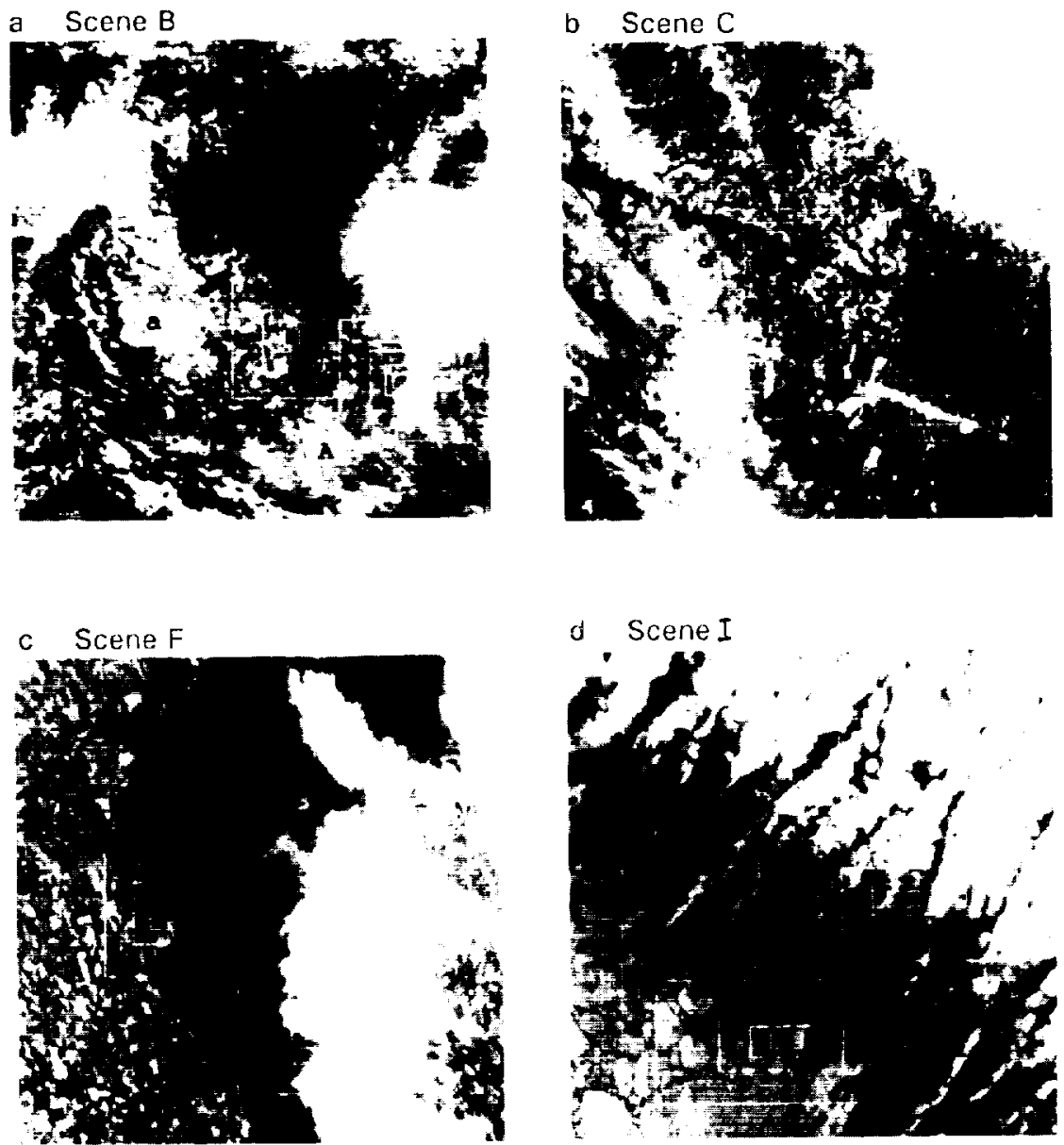

FiG. 13. The largest cloud templates are labeled as " $\mathrm{A}$ " and the largest corresponding shadow templates are labeled as "a." Each of the smaller templates is exactly one-half the length of the next larger template. Shown are (a) scene B; (b) scene C; (c) scene F; and (d) scene I.

matching approach to produce independent estimates of cirrus and stratus cloud heights. This is accomplished by applying a pattern matching approach using the cross-correlation function.

The method uses histogram equalization to expand the contrast in the scene and image inversion and fast Fourier transforms to improve the accuracy of the method. Through manual analysis, the cross-correlation method is shown to accurately match cloudshadow region pairs. The retrieved heights in four polar scenes are compared with temperature-based retrievals derived in mean monthly ECMWF analyses. In three of the four cases, good agreement is indicated. In a fifth comparison for a cirrus cloud over Texas, in which radiosonde soundings are available, reasonable agreement also is demonstrated. The effective azimuthal angle between the cloud and shadow templates is compared with the solar azimuth corrected for the orbital skew angle. This comparison is used as an indirect measure of retrieval accuracy, since no direct measure- ments are available. It is concluded that the retrieval method is accurate to within several pixels, and it is a function of cloud type and solar zenith angle. However, interpretation of the results is required to apply meaning to the retrieved height with respect to cloud base or top. Matched cloud-shadow pairs from the sunside correspond to a retrieved cloud-base height, while those from the antisunside correspond to a retrieved midlevel or top height. For low $(<2500 \mathrm{~m}$ ) clouds and large solar zenith angles $\left(>60^{\circ}\right)$ the technique is accurate to less than $100 \mathrm{~m}$. However, for high $(>10$ $\mathrm{km})$, transparent cirrus clouds at smaller solar zenith angles (e.g., $40^{\circ}$ ), the accuracy is on the order $1 \mathrm{~km}$. This and other cloud-shadow matching techniques have limitations in that they can only be applied to daytime imagery. In addition, without high spatial resolution elevation models, topography elevational differences between the surface beneath the cloud and the shadow introduce errors. And finally, matched cloudshadow pairs must be apparent and discernible. 
TABI.E 4. Retrieved cloud heights and azimuth errors as a function of spatial resolution for selected scenes.

\begin{tabular}{ccr}
\hline $\begin{array}{c}\text { Spatial resolution } \\
(\mathrm{m})\end{array}$ & $\begin{array}{c}\text { Cloud height } \\
(\mathrm{m})\end{array}$ & Azimuth error \\
\hline $\begin{array}{c}\text { Scene E } \\
57\end{array}$ & 7165 & \\
114 & 7243 & 0.1 \\
171 & 7243 & 0.2 \\
228 & 8063 & 0.2 \\
Scene I & & 12.0 \\
28.5 & 2388 & \\
57 & 2414 & 0.3 \\
85.5 & 2439 & 0.3 \\
114 & 2381 & 0.5 \\
142.5 & 2482 & -0.6 \\
171 & 2388 & 0.3 \\
199.5 & 2398 & 0.5 \\
228 & 2396 & -0.6 \\
285 & 2482 & 0.7 \\
342 & 2544 & 0.3 \\
399 & 2348 & -0.6 \\
456 & 2464 & 1.1 \\
& & 1.5 \\
\hline
\end{tabular}

The results show that precise location of the cloud templates is unnecessary. This is a positive result that suggests that an operational cirrus and stratus cloud height retrieval algorithm may be constructed. A semiautomated approach would require the reliable classification of cirrus and stratus cloud regions. However, based upon high spatial resolution cloud classification results by Kuo et al. (1988), Welch et al. (1989), Chen et al. (1989), and Lee et al. (1990), an effective global cloud masking scheme now is being developed and is scheduled to be used with the EOS Advanced Spaceborne Thermal Emission and Reflection Radiometer (ASTER) data. The results also show that the cloud templates of 64,128 , or 256 pixels on a side all produce equivalent cloud-height retrievals. However, templates of 16 and 32 pixels on a side are undesirable. It appears that a template of about 128 pixels on a side may be optimal. For areas in which there is considerable cloud cover, it is advisable to apply the smaller templates.

The results show that this method is fully applicable to the polar regions. In fact, the large solar zenith angles in the polar regions produce large shadow regions that improve the cloud-shadow matches. The application of this technique to polar regions is especially important due to the sparsity of sounding data there.

One objective of this study is to determine if this approach can be used with $250-\mathrm{m}$ spatial resolution MODIS data. The results suggest that spatial resolutions of about $150-200 \mathrm{~m}$ still produce reliable cloudheight retrievals, but that the method breaks down for spatial resolutions greater than $200 \mathrm{~m}$. Additional analysis is required to verify these preliminary findings. While the intent of the present investigation has been to demonstrate a potentially useful independent cloudheight retrieval scheme, follow-up studies will focus upon validation of the technique and its application to multilayer cloudiness.

Acknowledgments. Two of us (RMW and REF) have been supported by the National Aeronautics and Space Administration (NASA) Earth Observing System (EOS) contract NAS5-31718, and the other one of us (YI) is on a sabbatical by the Education Ministry of the Japanese Government. Appreciation is extended to three anonymous reviewers for their helpful suggestions, to Todd Berendes for two figures, and to Joie Robinson and Connie Crandall for typing the manuscript.

\section{REFERENCES}

Berendes, T., S. K. Sengupta, R. M. Welch, B. A. Wielicki, and M. Navar, 1992: Cumulus cloud base height estimation from high spatial resolution Landsat data: A Hough transform approach. IEEE Trans. Geosci. Remote Sens., 30, 430-443.

Chen. D. W., S. K. Sengupta, and R. M. Welch, 1989: Cloud field classification based upon high spatial resolution textural features. Part 2: Simplified vector approaches. J. Geophys. Res., 94(DI2), $14749-14765$.

Frouin, R., C. Gautier, and J. J. Morcrette, 1988: Downward longwave irradiance at the ocean surface from satellite data: Methodology and in situ validation. J. Geophys. Res., 93, 597-619.

Gonzalez, R. C., and P. Wintz, 1987: Digital Image Processing. 2d ed. Addison-Wesley, $503 \mathrm{pp}$.

Gupta, S. K., 1989: A parameterization for longwave surface radiation from sun-synchronous satellite data. J. Climate, 2, 305320

Gurney, C. M., 1982: The use of contextural information to detect cumulus clouds and cloud shadows in Landsat data. Int. J. Remote Sens., 3, 51-62.

Hahn, C. J., S. G. Warren, L. London, R. M. Chervin, and R. Jenne, 1982: Atlas of simultaneous occurrence of different cloud types over the ocean. NCAR Tech. Note, NCAR/TN-201, $212 \mathrm{pp}$.

Hambrick, L. N., M. H. Loew, and R. L. Carroll Jr., 1987: The entryexit method of shadow boundary segmentation. IEEE Trans. Patt. Anal. Mach. Intell., PAMI-9, 597-607.

King, M. D., Y. J. Kaufman, W. P. Menzel, and D. Tanre, 1992 Remote sensing of cloud, aerosol, and water vapor properties from the moderate resolution imaging spectrometer (MODIS). IEEE Trans. Geosci. Remote Sens., 30, 2-26.

Kuo, K. S., R. M. Welch, and S. K. Sengupta, 1988: Structural and textural characteristics of cirrus clouds observed using high spatial resolution Landsat imagery. J. Appl. Meteor., 27, 12421260.

- - R. C. Weger, M. A. Engelstad, and S. K. Sengupta, 1993: The three-dimensional structure of cumulus clouds over the ocean. Part I: Structural analyses. J. Geophys. Res., 98(D11), $20685-20711$.

Lee, J., R. Weger, S. K. Sengupta, and R. M. Welch, 1990: A neural network approach to cloud classification. IEEE Trans. Geosci. Remote Sens., 28, 846-855.

Manschke, A., 1988: Remote sensing of cloud base temperature in convective situations. Proc. Int. Radiation Symp. in Lille. International Association of Meteorology and Atmospheric Physics Radiation Commission, A. Deepak Publishing, $252 \mathrm{pp}$.

Mather. P. M., 1987: Computer Processing of Remotely-Sensed Images. John Wiley and Sons, $352 \mathrm{pp}$.

Minnis, P., K.-N. Liou, and Y. Takano, 1993: Inference of cirrus cloud properties using satellite-observed visible and infrared radiances. Part I: Parameterization of radiance fields. J. Atmos. Sci. 50, 1279-1304.

Mitchell, J. F. B., C. A. Senior, and W. J. Ingram, 1989: $\mathrm{CO}_{2}$ and climate: A missing feedback? Nature, 34, 132-134. 
Orheim, O., and B. K. Lucchitta, 1988: Numerical analysis of Landsat Thematic Mapper images of Antarctica: Surface temperatures and physical properties. Ann. Glaciol., 11, 109-120.

Pandey, P. C., E. G. Njoku, and J. W. Waters, 1983: Inference of cloud temperature and thickness by microwave radiometry from space. J. Climate Appl. Meteor., 22, 1844-1898.

Richards. J. A.. 1993: Remote Sensing Digital Image Analysis. $2 \mathrm{~d}$ ed. Springer-Verlag, $340 \mathrm{pp}$.

Robinove, C. J., 1982: Computation with physical values from Landsat digital data. Photogramm. Eng. Remote Sens., 48, 781-784.
Roeckner, E., U. Schlese, J. Biercamp, and P. Loewe, 1987: Cloud optical depth feedbacks and climate modeling. Nature, 329, $138-140$.

Welch, R. M., M. S. Navar, and S. K. Sengupta, 1989: The effect of spatial resolution upon texture-based cloud field classifications. J. Geophys. Res., 94(D12), 14 767-14 781.

Wu, M.-L. C. 1987: Determination of cloud ice water content and geometrical thickness using microwave and infrared radiometric measurements. J. Climate Appl. Meteor., 26, 878884 . 